\title{
Vibration modes and equivalent models for flexible rocking structures
}

\author{
Sinan Acikgoz ${ }^{1, *}$, , Matthew J. DeJong ${ }^{1}$ \\ ${ }^{1}$ Department of Engineering, University of Cambridge, Cambridge, UK
}

\begin{abstract}
Predicting the displacement and force response of flexible rocking structures to ground motion is important for their assessment and design. Insofar as practical, it is desirable to use simple mechanical models to make these predictions. However, the complex coupling between rocking and vibration makes accurate predictions with simple models difficult. In this paper, the use of semi-coupled equivalent models to approximate the dynamic response of multi-mass structures rocking on rigid ground is evaluated. These equivalent models feature a two-degree of freedom coupled rocking oscillator to describe the interaction of rocking and the first mode of vibration, and uncoupled linear elastic oscillators to describe higher mode vibration response. To evaluate these equivalent models, the modal components of the dynamic response of multi-mass structures are first determined. These components highlight the critical influence of the excitation of vibration modes at impact. Then, further investigations are carried out by comparing equivalent model simulations to recent shake table tests and multi-mass analytical model simulations. These comparisons reveal that the equivalent models can capture the rocking response accurately for a realistic range of displacements, if a new ground acceleration scaling term is adopted. However, the uncoupled linear elastic oscillators do not consider excitation at impact and consequently, the equivalent models do not capture the acceleration response adequately. Therefore, on the basis of the analytically identified modal components, a further modification that improves the equivalent model acceleration predictions is proposed and validated.
\end{abstract}

KEY WORDS: modal analysis; equivalent model; rocking; uplift; shake table

\footnotetext{
* Correspondence to: Sinan Acikgoz, Department of Engineering, University of Cambridge, Trumpington Street, Cambridge, CB2 1PZ, UK

§ E-mail: msa44@cam.ac.uk
} 


\section{INTRODUCTION}

There is a growing interest in using rocking mechanisms for the earthquake resistant design of structures (Mander and Cheng 1997; Priestley et al. 1999; Palermo et al. 2005; Toranzo et al. 2009; Pollino and Bruneau 2010; Marriott et al. 2011; Wiebe et al. 2012a; Wiebe et al. 2012b). In particular, base rocking mechanisms have been implemented in several building (Kelly and Tsztoo 1977; Huckelbridge and Clough 1978; Sharpe and Skinner 1983) and bridge systems (Beck and Skinner 1973; Cheng 2007). Predicting the displacement and force response of these structures to strong ground motions is essential for their assessment and design.

In earthquake engineering analysis and design, single degree of freedom (SDOF) linear elastic oscillators are extensively used for determining the earthquake response of multi-mass and multi-degree of freedom elastic structures which are fixed to the ground (Chopra 2001). In this method, the earthquake response is described as the superposition of uncoupled SDOF oscillators. This simplification facilitates analysis and design by reducing the complexity of the investigated mechanical systems, thereby focussing on the critical response parameters. In an analogous fashion, the objective of this paper is to improve simple mechanical models that describe the rocking and acceleration response of complex systems, and to evaluate the effectiveness of these models by comparison to the results of physical experiments and computational simulations which employ multi-mass flexible rocking models.

Pioneering studies on the dynamics of flexible rocking systems investigated idealized models with one or two lumped masses (Meek 1975; Chopra and Yim 1985; Psycharis 1991). These studies describe structures which are allowed to vibrate and rock freely on a rigid surface. They clearly demonstrate that vibrations and rocking action are coupled; once rocking initiates, vibration frequencies and excitation mechanisms change. In return, vibrations cause oscillatory rocking action. Due to this prominent coupling, the equations of motion describing vibration and rocking response need to be solved simultaneously. Therefore, reducing the system to a superposition of uncoupled single degree of freedom systems is challenging. Later studies quantified the significant effects of this coupling on salient response characteristics, such as maximum base shear and overturning stability (Oliveto et al. 2003; Acikgoz and DeJong 2012; Acikgoz and DeJong 2013b; Acikgoz and DeJong 2013c; Vassiliou et al. 2015).

Analytical investigations of more complex flexible rocking structures with many degrees of freedom (Meek 1978; Psycharis 1983; Yim and Chopra 1985) similarly showed that the lowest vibration mode interacts strongly with rocking action. In contrast, for the higher vibration modes, the coupling was deemed negligible. On the basis of this assumption, simpler 'equivalent models' which describe the earthquake response of multimass structures were proposed (Yim and Chopra 1985). These superpose the response of a coupled two degree of freedom model and uncoupled SDOF oscillators. The coupled two degree of freedom model describes the combined response of the first vibration mode and rocking action. The uncoupled SDOF oscillators superpose the higher vibration mode responses which are assumed not to interact with rocking.

The proposed equivalent models can considerably simplify the analysis and design of multi-mass flexible rocking structures. However, these models need further investigation and validation with experiments. Therefore, the modal components of the dynamic response of multi-mass structures are first examined in Section 2. This new information allows a critical evaluation of the derivation of equivalent models, which follows the assumptions set out in earlier work (Yim and Chopra 1985) (see Section 3). Then, the proposed models' equivalence to complex multi-mass flexible rocking models is investigated with 
comparisons to multi-mass analytical model simulations and recent shake table tests. Here, the accuracy of the equivalent models in predicting the rocking and acceleration response is quantified. On the basis of these results, modified equivalent models are proposed to better capture the overturning moments due to ground accelerations and the excitation of higher vibration modes at impact. Finally, Section 5 summarizes the conclusions from this study.

\section{MODAL COMPONENTS OF FLEXIBLE ROCKING RESPONSE}

In this study, equivalent models will be derived to approximate the dynamic behaviour of complex flexible rocking structures. Therefore, a general analytical model of a multi-mass flexible rocking structure with many degrees of freedom (Acikgoz and DeJong 2016) will first be examined. Also in this section, the modal components of this model are analytically determined to identify salient dynamic characteristics.

\subsection{Review of the multi-mass analytical model}

The multi-mass flexible rocking model can describe a wide range of structures, including elastic building frames (Acikgoz and DeJong 2013a) and tower structures (Acikgoz et al. 2014). The linearized equations of motion of this model are reviewed in this section. The model is illustrated in Figure 1 during the full contact (left) and rocking phases (right). It features $N+1$ nodes, where masses are lumped. At node $N$ (situated at height $H_{N}$ ), the mass is denoted by $m_{N}$. Node 0 is located at $H_{0}=0$, with lumped mass $m_{0}$ and rotational inertia $J_{0}$. This node is restrained from participating in elastic motion. For nodes 1 to $N$, the $N \times 1$ vector $\underline{H}$ describes nodal heights and $N \times N$ diagonal mass matrix $\mathbf{M}$ describes the nodal masses. The two degrees of freedom (DOF) associated with the small elastic lateral translation and rotation are illustrated by $u_{N}$ and $\Omega_{N}$ for node $N$ in Figure 1 . For nodes 1 to $N$, these DOFs can be described in vector notation as $\underline{u}$ and $\underline{\Omega}$. For the examined structures, the elastic rotation degree of freedom can be statically condensed. Therefore, the stiffness of this structure during full contact can be described by the $N \times N$ condensed stiffness matrix $\mathbf{K}$. Using the mass and stiffness matrices, a $N \times N$ proportional damping matrix $\mathbf{C}$ can also be defined.

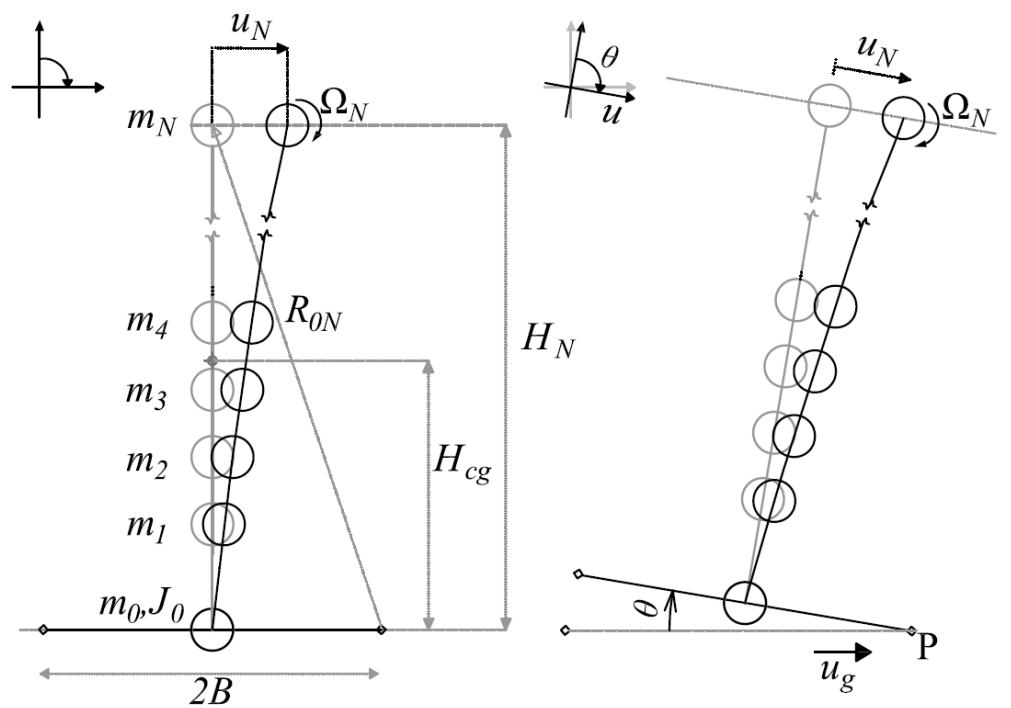

Figure 1 - Schematic of the multi-mass analytical model during the full contact (top left) and rocking (top right) phases 
During the full contact phase, the following equation of motion describes the dynamic force equilibrium in the direction of $\underline{u}$ :

$$
\mathbf{M} \underline{\ddot{u}}+\mathbf{C} \underline{\dot{u}}+\mathbf{K} \underline{u}+\mathbf{M} \underline{1} \ddot{u}_{g}=0
$$

where a dot above the variables indicates differentiation with respect to time $t$ and 1 specifies an $N \times 1$ vector of ones.

Once conditions required for uplift are met, the system progresses to the rocking phase. During the rocking phase, the structure rotates about pivot points located at the edges of the foundation (see Figure 1, right). These pivot points are a distance of $2 B$ apart from one another and lie at radial distances of $R_{0}$ from nodes. Rocking occurs at the interface of the rigid foundation beam and rigid ground while maintaining contact with the current pivot point $\mathrm{P}$. The parameter $\theta$ describes the rocking action. Two sets of equations describe the dynamic equilibrium. $N$ equations describe dynamic force equilibrium in the direction of $\underline{u}$ for nodes 1 to $N$ and one equation describes dynamic moment equilibrium about the current pivot point $P$. Linearization of these equations about the static at rest configuration ( $\theta=0, \dot{\theta}=0, \underline{u}=\underline{0}$ and $\underline{\dot{u}}=\underline{0})$ yields:

$$
\begin{gathered}
\mathbf{M} \underline{\ddot{u}}+\mathbf{M} \underline{H} \ddot{\theta}+\mathbf{C} \underline{\dot{u}}+\mathbf{K} \underline{u}-\mathbf{M} \underline{1} g \theta+\mathbf{M} \underline{1} \ddot{i}_{g}=\underline{0} \\
\underline{H}^{T} \mathbf{M} \underline{\ddot{u}}+J_{\mathrm{p}} \ddot{\theta}+g\left(-\underline{u}^{T} \mathbf{M} \underline{1}-\underline{H}^{T} \mathbf{M} \underline{1} \theta\right)+m_{t} H_{c g} \ddot{u}_{g} \pm m_{t} g B=0
\end{gathered}
$$

where $g$ denotes gravitational acceleration, $m_{t}$ denotes the total mass of the structure, $H_{c g}$ is the height of centre of gravity, and the system mass moment of inertia about the pivot point $\mathrm{P}$ is denoted by $J_{\mathrm{P}}=R_{0}{ }^{T} \mathbf{M} R_{0}+m_{0} B^{2}+J_{0}$. In these equations, the upper sign refers to rocking about the right pivot point and the lower sign refers to rocking about the left pivot point. A similar notation will be used throughout this paper to describe the piecewise defined equations of motion.

Equations 2-3 are a set of second order differential equations with constant coefficients. Therefore, they may be expressed in the following format:

$$
\mathbf{M}_{\mathbf{L}} \underline{\ddot{z}}+\mathbf{C}_{\mathbf{L}} \underline{\dot{z}}+\mathbf{K}_{\mathbf{L}} \underline{z}=-\underline{F_{L}}
$$

where the state vector $\underline{z}=[\underline{u}, \theta]$ encompasses all the variables in the system. The global mass, damping and stiffness matrices are defined respectively as $\mathbf{M}_{\mathbf{L}}, \mathbf{C}_{\mathbf{L}}$ and $\mathbf{K}_{\mathbf{L}} \cdot F_{L}$ is the forcing vector.

Full contact response $\approx 1^{\text {st }}$ vibration mode $+2^{\text {nd }}$ vibration mode $+3^{\text {rd }}$ vibration mode

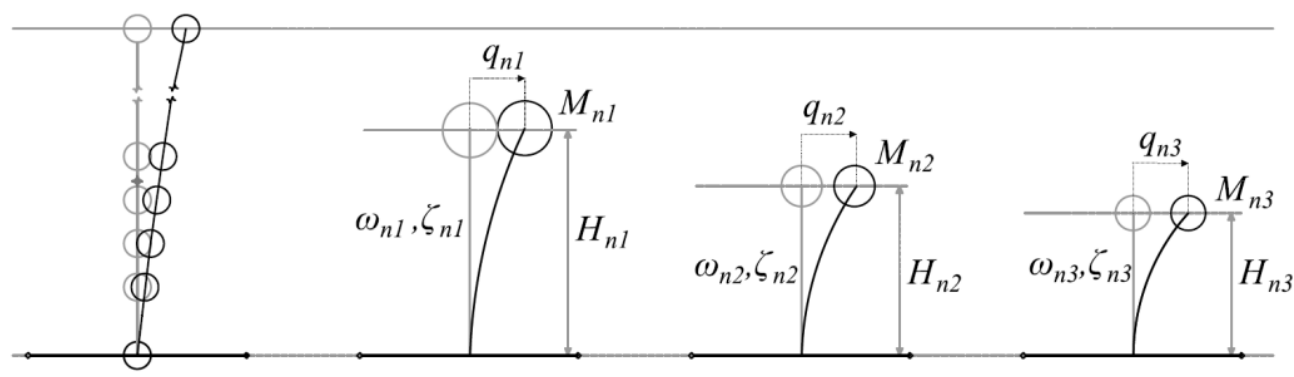

Figure 2 - Schematic description of the modal components of the multi-mass analytical model response during the full contact phase 


\subsection{Modal components of response}

\section{Full contact phase}

When the damping matrix $\mathbf{C}$ is proportional, it is possible to decouple Equation 1 into its modal components (see Figure 2). To do this, the state vector, $\underline{u}$ can be expressed using Rayleigh's method as:

$$
\underline{u}=\boldsymbol{v}_{\mathbf{n}} \underline{q_{n}}
$$

where $\mathbf{v}_{\mathbf{n}}=\left[\underline{v_{n 1}}, \underline{v_{n 2}}, \ldots, \underline{v_{n N}}\right]$ is the matrix of eigenvectors, where the $j^{\text {th }}$ column of $\boldsymbol{v}_{\mathbf{n}}$ describes the $j^{\text {th }}$ eigenvector of the system and is denoted by $v_{n j}$, and the generalized coordinate vector $\underline{q_{n}}$ describes the modal solutions as follows:

$$
\underline{q_{n}}=\left(q_{n 1}, q_{n 2}, \ldots ., q_{n N}\right)^{T}=\left(\varepsilon_{n 1} e^{\Lambda_{n 1} t}, \varepsilon_{n 2} e^{\Lambda_{n 2} t}, \ldots ., \varepsilon_{n N} e^{\Lambda_{n N} t}\right)^{T}
$$

where $\varepsilon_{n j}$ is a scale factor describing the time-varying response of the $j^{\text {th }}$ mode, and $\Lambda_{n j}$ denotes the eigenvalue of the $j^{\text {th }}$ normal mode of the system. Using this notation, the homogenous form of Equation 1 becomes:

$$
\left(\mathbf{K}+\Lambda_{n j}{ }^{2} \mathbf{M}\right) \underline{v_{n j}} \varepsilon_{n j} e^{\Lambda_{n j} t}=\underline{0}
$$

The eigenvectors and eigenvalues can be obtained by solving Equation (7). Here, the vibration mode $j$ is uncoupled from other vibration modes due to modal orthogonality. This suggests that for two arbitrary vibration modes $j$ and $k$, where $j \neq k$ and $\Lambda_{n j} \neq \Lambda_{n k}$, the following mass and stiffness orthogonality relationships are applicable:

$$
\underline{v_{n j}}{ }^{T} \mathbf{M} \underline{v_{n k}}=0, \underline{v_{n j}}{ }^{T} \mathbf{K} \underline{v_{n k}}=0
$$

The $j^{\text {th }}$ modal component of response during full contact stage can be expressed as:

$$
\underline{v_{n j}{ }^{T}} \mathbf{M} \underline{v_{n j}} \ddot{q}_{n j}+\underline{v_{n j}}{ }^{T} \mathbf{C} v_{n j} \dot{q}_{n j}+\underline{v_{n j}}{ }^{T} \mathbf{K} v_{n j} q_{n j}+\underline{v_{n j}}{ }^{T} \mathbf{M} \underline{1} \ddot{i}_{g}=0
$$

Equation 9 can be simplified by expressing it in terms of modal vibration frequency and damping. These components are respectively described as follows:

$$
\omega_{n j}{ }^{2}=\frac{v_{n j}{ }^{T} \mathbf{K} v_{n j}}{\underline{v_{n j}{ }^{T}} \mathbf{M} \underline{v_{n j}}}, 2 \zeta_{n j} \omega_{n j}=\frac{v_{n j}{ }^{T} \mathbf{C} v_{n j}}{\underline{v_{n j}{ }^{T}} \mathbf{M} \underline{v_{n j}}}
$$

In addition, the effective modal mass and effective modal height are also defined here for reference:

$$
M_{n j}=\frac{\left(v_{n j}{ }^{T} \mathbf{M} \underline{1}\right)^{2}}{\underline{v_{n j}{ }^{T}}{ }^{\mathbf{M}} \underline{v_{n j}}}, H_{n j}=\frac{{\underline{v_{n j}}}^{T} \mathbf{M} \underline{H}}{\underline{v_{n j}{ }^{T}} \mathbf{M} \underline{1}}
$$

Note that all of the modal parameters in Equations (10-11) are independent of the arbitrary scaling of the eigenvector. To simplify the resulting equations, the eigenvectors can be scaled such that $\frac{v_{n j}{ }^{T} \mathbf{M} 1}{{\underline{v_{n j}}}^{T} \mathbf{M} \underline{v}_{n j}}=1$. Equation 9 then simply becomes the equation of a single degree of freedom system: 


$$
\ddot{q}_{n j}+2 \zeta_{n j} \omega_{n j} \dot{q}_{n j}+\omega_{n j}{ }^{2} q_{n j}+\ddot{u}_{g}=0
$$

After determining $q_{n j}$ for $j=1, \ldots, N$, the total response of the coupled system during full contact phase may be calculated by using Equation (5).

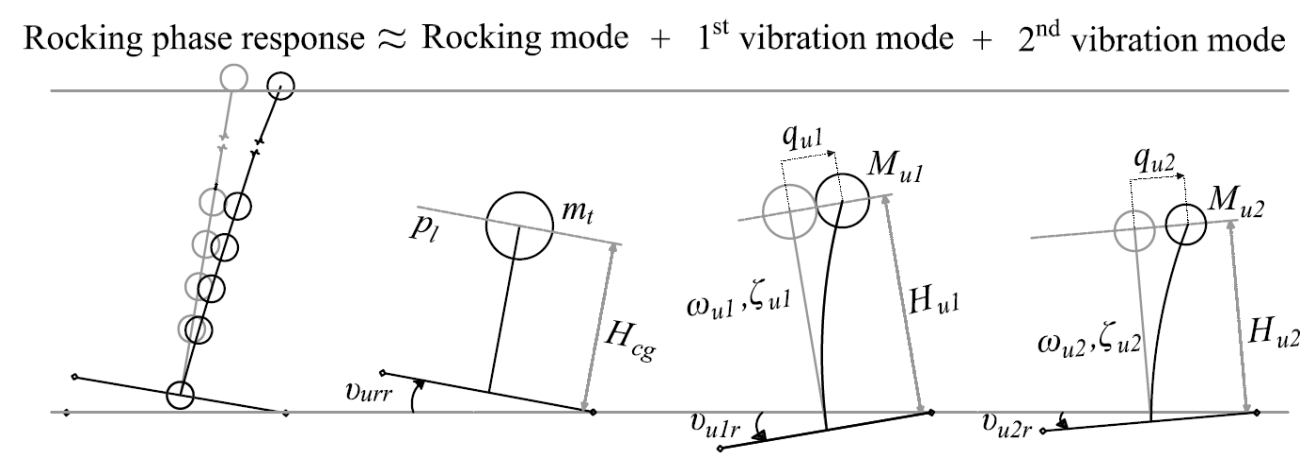

Figure 3 - Schematic description of the modal components of the multi-mass analytical model response during the rocking phase

\section{Rocking phase}

Using a similar approach, the eigen-parameters of Equations 2 and 3 can be found to decompose the response into modal components (see Figure 3). Assuming a proportional damping matrix $\mathbf{C}_{\mathbf{L}}$, the state vector may be expressed as follows:

$$
\underline{z}=v_{\mathbf{u}} \underline{q_{u}}
$$

where $\boldsymbol{v}_{\mathbf{u}}=\left[\underline{v_{u 1}}, \underline{v_{u 2}}, \ldots, \underline{v_{u(N+1)}}\right]$ is the $(N+1) \times(N+1)$ matrix of rocking phase eigenvectors. The $j^{\text {th }}$ column of $\boldsymbol{v}_{\mathrm{u}}$ describes the $j^{\text {th }}$ eigenvector of the system, denoted by $v_{u j}$ and is composed of (i) ${\underline{v_{u j t}}}_{\text {, }}$, the elastic eigenvector component in the direction of $u$ and (ii) $v_{u j r}$, the rocking component which describes the rigid body rotation of the structure as a whole:

$$
\underline{v_{u j}}=\left(\underline{v_{u j t}{ }^{T}}, v_{u j r}\right)^{T}
$$

The generalized coordinate vector $q_{u}$ is given by:

$$
\underline{q_{u}}=\left(q_{u 1}, q_{u 2}, \ldots ., q_{u N}, q_{u N+1}\right)^{T}=\left(\varepsilon_{u 1} e^{\Lambda_{u 1} t}, \varepsilon_{u 2} e^{\Lambda_{u 2} t}, \ldots . ., \varepsilon_{u N} e^{\Lambda_{u N} t}, \varepsilon_{u N+1} e^{\Lambda_{u N+1} t}\right)^{T}
$$

where $q_{u j}$ denotes the generalized coordinate for mode $j=1, \ldots, N+1$. This coordinate is characterized by the eigenvalue $\Lambda_{u j}$ and the scaling factor $\varepsilon_{u j}$. Assuming a solution of the form $\underline{z}=\underline{v_{u j}} \varepsilon_{u j} e^{\Lambda_{u j} t}$ and substituting this into the homogenous form of Equation (4), yields:

$$
\left(\mathbf{K}_{\mathbf{L}}+\Lambda_{u j}{ }^{2} \mathbf{M}_{\mathbf{L}}\right) \underline{v_{u j}} \varepsilon_{u j} e^{\Lambda_{u j} t}=\underline{0}
$$

Since the mass and stiffness matrices $\mathbf{M}_{\mathbf{L}}$ and $\mathbf{K}_{\mathbf{L}}$ are real-valued and symmetric, each mode is expressed by a complex conjugate eigenvalue couple. When these eigenvalues are distinct, the modes are orthogonal. From Equation (16), the unscaled real eigenvectors $v_{u j}$ corresponding to normal modes may be determined to derive modal equations. 
Alternatively, an analytical estimation of the modal equations can be achieved when corresponding eigenvectors can be estimated. Accumulating experimental and analytical evidence (Acikgoz and DeJong 2013a; Acikgoz and DeJong 2013b; Acikgoz et al. 2014; Acikgoz et al. 2016; Acikgoz and DeJong 2016) suggest that the dynamic response of a flexible rocking structure is composed of: (i) one weakly coupled rocking mode and (ii) $N$ coupled vibration modes (see Figure 3 for an illustration of these modes which feature both vibration and rocking components), where the degree of coupling varies. Within this context, strong and weak coupling refer to the degree of influence of superstructure vibrations on the characteristics of rocking response (such as frequency) and vice versa. For instance, the rocking mode describes the rigid body rotation of the structure as a whole with negligibly small elastic motion. Therefore the coupling is weak and a rocking mode eigenvector $v_{u r}$ (denoted by mode subscript $r$ ) can be reliably defined where the elastic component $v_{u r t}=\underline{0}$ and rocking component $v_{u r r}=1$. Assuming modal orthogonality, premultiplying Equation 4 by this eigenvector yields:

$$
\ddot{q}_{u r}-p_{l}^{2} q_{u r}+p_{l}^{2} \frac{\ddot{u}_{g}}{g} \pm p_{l}^{2} \tan \left(\alpha_{c g}\right)=0
$$

where the generalized coordinate vector $q_{u r}$ describes the rocking mode solution. The eigenvalue $p_{l}$ is the well-known frequency parameter, which provides a linear approximation of the pendulum frequency of the structure as if it were suspended from its pivot point. It is given by:

$$
p_{l}=\sqrt{\frac{\underline{1}^{T} \mathbf{M} \underline{H} g}{J_{\mathrm{P}}}}
$$

It is noteworthy that the Equation (17) is identical to the dynamic moment equilibrium equation of a rigid block, linearized about $\theta=0$.

The equations for the remaining $N$ coupled vibration modes can be determined in a similar manner. However, it is difficult to determine the mode shapes a priori. Recent work by the authors (Acikgoz and DeJong 2016) suggests that the eigenvectors for the coupled vibration modes could be distinct from the eigenvectors during full contact stage due to coupling. Therefore, an alternative approach is adopted here. For the arbitrary vibration mode $k$ of the structure during rocking, the rocking component of the eigenvector $v_{u k r}$ is estimated in terms of the elastic component of the same vibration mode $\underline{v_{u k t}}$. Considering Equations (3) and (17), and neglecting geometric stiffness due to small elastic deformations, it is postulated that all orthogonal modes which involve coupled rocking and vibration action need to satisfy dynamic moment equilibrium around the pivot point. This implies that, for any vibration mode $k$, where $k=1, \ldots, N$ :

$$
v_{u k r}=\frac{-\underline{H}^{T} \mathbf{M} \underline{v k t}}{J_{\mathrm{P}}}
$$


Then, by premultiplying Equation 4 by the eigenvector of mode $k, \underline{v_{u k}}=\left(\underline{v_{u k t}}, \frac{-\underline{H}^{T} \mathbf{M} \underline{u k t}}{J_{\mathrm{P}}}\right)^{T}$, the equations for the coupled vibration modes can be obtained. For convenience, $\underline{v}_{u k}$ is scaled such that $\frac{\underline{v u k t}_{u} \mathbf{M} 1}{\underline{v_{u k}} \mathbf{M}_{\mathbf{L}} \underline{v_{u k}}}=1$, to yield:

$$
\ddot{q}_{u k}+2 \zeta_{u k} \omega_{u k} \dot{q}_{u k}+\omega_{u k}^{2} q_{u k}+f_{u k}=0
$$

where modal frequency $\omega_{u k}$, modal damping $\zeta_{u k}$ and modal forcing $f_{u k}$ of the $k$ vibration modes are introduced. It is noteworthy that Equation (20) is a SDOF oscillator equation, similar to full contact phase modal components in Equation (12). However, the modal characteristics of the system in Equation (20) are distinct. The effective modal masses and the effective height of the vibration modes during rocking can be introduced in analogy with Equation (11):

$$
M_{u k}=\frac{\left({\underline{v_{u k t}}}^{T} \mathbf{M} \underline{1}\right)^{2}}{\underline{v_{u k t}{ }^{T}}{ }^{M} \underline{v_{u k t}}}, H_{u k}=\frac{{\underline{v_{u k t}}}^{T} \mathbf{M} \underline{H}}{\underline{v_{u k t}}{ }^{T} \mathbf{M} \underline{1}}
$$

The premultiplying operation which yields Equation (20) results in the following modal frequency equation:

$$
\omega_{u k}^{2}=\frac{\frac{v_{u k t}^{T} \mathbf{K}{\underline{v_{u k t}}}^{T} \mathbf{M} v_{u k t}}{v_{l} \chi_{u k}}}{1-\chi_{u k}}
$$

where $\chi_{u k}=\frac{M_{u k} H_{u k}{ }^{2}}{J_{p}}$ is the modal mass participation factor. Equation (22) expresses the modal frequency in terms of the full contact phase mass and stiffness matrices and provides insight into the behaviour of coupled vibration modes, even without prior knowledge of the eigenvector $v_{u k}$. Earlier studies (Psycharis 1983; Yim and Chopra 1985) suggested that the classical eigenvalues and eigenvectors identified from Equation (7) for the full contact phase would be valid during the rocking phase for all vibration modes except the lowest. This would imply that $\underline{v_{u k t}}=\underline{v_{n k}}$ for $k=2, \ldots, N$. However, Equation (22) suggests that, despite the assumption of identical eigenvectors, rocking phase eigenvalues would be distinct from full contact phase eigenvalues when $\chi_{u k} \neq 0$. In these cases, as indicated by Equation (22), a change in vibration frequencies is expected with the initiation of rocking.

Similarly, a closed-form modal forcing function $f_{u k}$ can be obtained with the premultiplying operation which yields Equation (20). It is given by:

$$
f_{u k}=\left(1-l_{u k}\right) \ddot{u}_{g} \mp\left(\imath_{u k}\right) g \tan \left(\alpha_{c g}\right)
$$

where the new force modification parameter $\imath_{u k}$ is introduced:

$$
l_{u k}=\frac{p_{l}^{2} H_{u k}}{g}
$$


Equations (23) and (24) highlight further aspects concerning the coupled vibration modes. Alongside changes in the vibration frequency (see Equation (22)), the coupling of vibrations and rocking result in potential changes in the modal excitation mechanisms once uplift occurs. Contrasting the modal forcing terms during full contact and rocking phases in Equations (12) and (23), two aspects can be highlighted. The first is the apparent reduction of the ground acceleration forcing by the force modification parameter $t_{u k}$ in Equation (23). This suggests that for vibration modes with large effective height $H_{u k}$, and therefore large values of $t_{u k}$, superstructure vibrations will be partially isolated from the effects of ground motion. The second aspect is the introduction of a gravity forcing term $g \tan \left(\alpha_{c g}\right)$, which is negative for positive rocking angles and positive for negative rocking angles. This term is responsible for the strong excitation of vibrations at impact due to gravity force reversal and strongly depends on the force modification parameter $l_{u k}$. For vibration modes with large values of $v_{u k}$, gravity forcing will be significant. This counteracts the simultaneous isolation effect and needs to be accounted for while evaluating the force demands on the system.

In brief, the Equations (17) and (20) describe the independent modal components of rocking response and provide new insight into response. After determining $q_{u r}$ from Equation (17) and $q_{u k}$ from Equation (20) for $k=1, \ldots, N$, the total response of the coupled system during rocking may be calculated by using Equation (13). However, the equations of motion describing rocking motion are piecewise defined and terminate when the rocking structure impacts with the ground at $\theta=0$. Therefore, in order to accurately determine the time of impact, these modal equations need to be solved simultaneously. This shows that further simplifications are necessary to arrive at equivalent models.

\section{DERIVATION OF EQUIVALENT MODELS}

\subsection{Simplification of response}

In order to describe the complex multi-mass structures with the use of simple equivalent models, the assumptions proposed by Yim and Chopra (1985) are adopted in this section. This paper considers a structure rocking on a rigid base instead of the two-spring foundation considered in the original Yim and Chopra paper, and therefore the resulting equations are different. However, unless explicitly stated, the derivation approach is identical to the earlier work (Yim and Chopra 1985).

Yim and Chopra's (1985) approach relies on two major assumptions: (i) the classical vibration mode shapes identified during the full contact phase for the $2^{\text {nd }}$ and higher vibration modes are valid during rocking phase and (ii) the effective modal height of the $2^{\text {nd }}$ and higher vibration modes is zero. Therefore, as a first step, Equations (2) and (3) are expressed using the classical eigen-parameters identified using Equation (7). After neglecting geometric stiffness terms, the following equations are obtained:

$$
\begin{gathered}
\mathbf{M} v_{\mathbf{n}} \underline{\ddot{q}_{n}}+\mathbf{M} \underline{H} \ddot{\theta}+\mathbf{C} v_{\mathbf{n}} \underline{\dot{q}_{n}}+\mathbf{K} \mathbf{v}_{\mathbf{n}} \underline{q_{n}}+\mathbf{M} \underline{\ddot{u}_{g}}=\underline{0} \\
\underline{H^{T}} \mathbf{M} v_{\mathbf{n}} \underline{\ddot{q}_{n}}+J_{\mathrm{p}} \ddot{\theta}+m_{t} H_{c g} \ddot{u}_{g} \pm m_{t} g B=0
\end{gathered}
$$

Pre-multiplying Equation (25) by the classical vibration mode shape $\underline{v_{n j}{ }^{T}}$ and utilizing modal orthogonality relations in Equation (8) yields:

$$
\underline{v_{n j}{ }^{T} \mathbf{M}} \underline{v_{n j}} \ddot{q}_{n j}+\underline{v_{n j}{ }^{T}} \mathbf{M} \underline{H} \ddot{\theta}+\underline{v_{n j}} \underline{\mathbf{C} v_{n j}} \dot{q}_{n j}+\underline{v_{n j}}{ }^{T} \mathbf{K}{\underline{v_{n j}}}_{n j}+\underline{v_{n j}}{ }^{T} \mathbf{M} \underline{1} \ddot{u}_{g}=0
$$


If the eigenvectors are scaled such that $\frac{v_{n j}{ }^{T} \mathbf{M} 1}{\underline{v_{n j}{ }^{T}}{ }^{M} \underline{v_{n j}}}=1$, Equation (27) becomes

$$
\ddot{q}_{n j}+H_{n j} \ddot{\theta}+2 \zeta_{n j} \omega_{n j} \dot{q}_{n j}+\omega_{n j}{ }^{2} q_{n j}+\ddot{u}_{g}=0
$$

Using the classical modal descriptions, Equation (26) becomes:

$$
\sum_{j=1}^{N} M_{n j} H_{n j} \ddot{q}_{n j}+J_{\mathrm{p}} \ddot{\theta}+m_{t} H_{c g} \ddot{u}_{g} \pm m_{t} g B=0
$$

Equation (28) is similar to the equation of a SDOF oscillator with frequency $\omega_{n j}$ and damping $\zeta_{n j}$. However, due to the presence of the mass coupling term $H_{n j} \ddot{\theta}$, this equation is not decoupled from Equation (29). Similarly, Equation (29) also demonstrates mass coupling, indicating that these equations need to be solved simultaneously for an exact solution. However, using the aforementioned assumptions, these equations can be further simplified. After assuming that the effective modal height $H_{n j}$ of $2^{\text {nd }}$ and higher vibration modes is zero just like Yim and Chopra (1985), Equation (28) and (29) effectively become the combination of a two-degree of freedom coupled system:

$$
\begin{gathered}
\ddot{q}_{n 1}+H_{n 1} \ddot{\theta}+2 \zeta_{n 1} \omega_{n 1} \dot{q}_{n 1}+\omega_{n 1}{ }^{2} q_{n 1}+\ddot{u}_{g}=0 \\
M_{n 1} H_{n 1} \ddot{q}_{n 1}+J_{\mathrm{p}} \ddot{\theta}+m_{t} H_{c g} \ddot{u}_{g} \pm m_{t} g B=0
\end{gathered}
$$

and the vibration equations of the higher modes, $k=2, \ldots, N$, which are uncoupled:

$$
\ddot{q}_{n k}+2 \zeta_{n k} \omega_{n k} \dot{q}_{n k}+\omega_{n k}^{2} q_{n k}+\ddot{u}_{g}=0
$$

The resulting Equation (32) is distinct from the coupled vibration mode equilibrium Equation (20). Equation (20) suggested that higher mode vibrations may also be excited by gravity forcing, and this external forcing is notably absent in Equation (32).

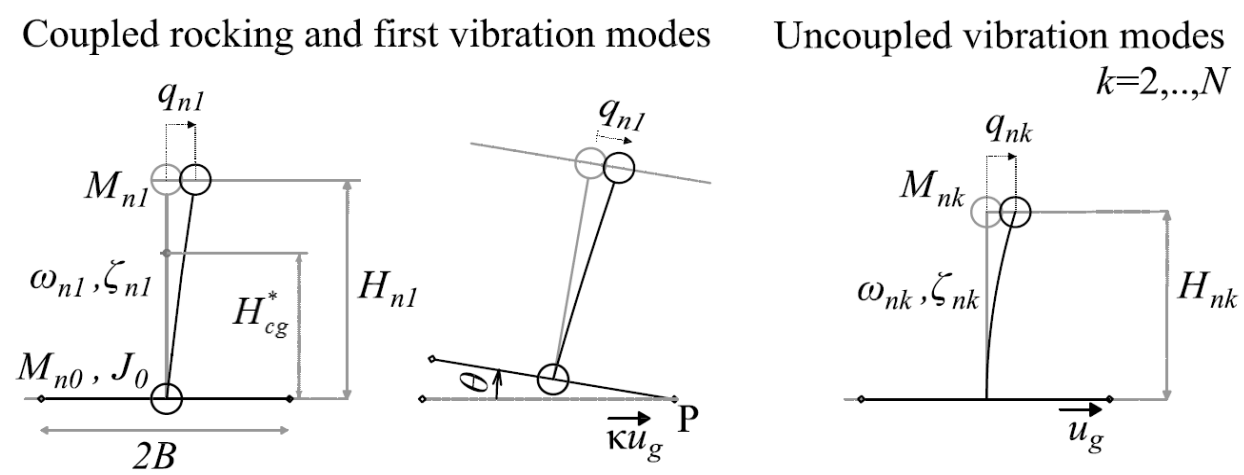

Figure 4 - Schematic description of the equivalent model, with the coupled rocking and first vibration mode components during full contact and rocking phase (left and middle) and the uncoupled vibration mode components (right)

\subsection{Input parameters}

The simplified Equations (30) and (31) describe a two degree of freedom coupled system. Combined with Equation (32), which describes the uncoupled higher vibration modes, this system of equations represents the multi-mass system. It is desirable to describe the coupled two degree of freedom component of the equivalent model with the physical parameters of a simple flexible rocking structure model with two lumped masses, such as 
the one presented in Figure 4 (left and middle). Therefore, in this section, parameters are defined to approximate Equations (30) and (31) with the physical two lumped mass model that is illustrated in Figure 4.

To do this, it is useful to compare Equations (30) and (31) to the actual equations of motion of a flexible rocking structure model with two lumped masses, as presented in Figure 4 (left and middle). The equations of motion for this two lumped mass model can be determined by plugging in the relative parameters to the general equations of motion, Equations (2) and (3). According to Figure 4, the parameters for the lumped top mass are mass $\mathbf{M}=M_{n 1}$ at height $\underline{H}=H_{n 1}$, vibration frequency $\omega_{n 1}$ and damping factor $\zeta_{n 1}$. These parameters are identical to the first vibration mode characteristics of the multi-mass model, and they are utilized as the superstructure characteristics of the two lumped mass model. The model in Figure 4 also has a bottom mass $M_{n 0}$ and an additional mass moment of inertia $J_{0}$. Using these parameters and neglecting the geometric stiffness terms in Equations (2) and (3), the equations of motion for the model in Figure 4 is obtained as follows:

$$
\begin{gathered}
\ddot{q}_{n 1}+H_{n 1} \ddot{\theta}+2 \zeta_{n 1} \omega_{n 1} \dot{q}_{n 1}+\omega_{n 1}^{2} q_{n 1}+\ddot{u}_{g}=0 \\
M_{n 1} H_{n 1} \ddot{q}_{n 1}+\left(M_{n 1} H_{n 1}^{2}+\left(M_{n 0}+M_{n 1}\right) B^{2}+J_{0}\right) \ddot{\theta}+\left(M_{n 0}+M_{n 1}\right) H_{c g}^{*} \ddot{u}_{g} \pm\left(M_{n 0}+M_{n 1}\right) g B=0
\end{gathered}
$$

where the height of centre of gravity of the model in Figure 4 is represented as $H_{c g}^{*}$. Equations (33) and (34) are similar to Equations (30) and (31). In fact, Equation (33) is identical to Equation (30). To make sure Equation (34) and (31) match, the values of bottom mass $M_{n 0}$ and additional mass moment of inertia $J_{0}$ need to be calibrated. The last term of Equation (31) describes the restraining moments provided by gravity as $m_{t} g B$, whereas Equation (34) describes these moments as $\left(M_{n 0}+M_{n 1}\right) g B$. Therefore the bottom mass $M_{n 0}$ may be determined as follows:

$$
M_{n 0}=m_{t}-M_{n 1}
$$

Recall that, $m_{t}$ was described earlier as the total mass of the multi-mass model. Similarly, the second term of Equations (31) and (34) describe the mass moment of inertia of the system about the pivot point. The multi-mass system has a total inertia of $J_{\mathrm{p}}$. Therefore, $J_{0}$ may be determined as follows:

$$
J_{0}=J_{\mathrm{p}}-\left(M_{n 0}+M_{n 1}\right) B^{2}-M_{n 1} H_{n 1}^{2}
$$

Finally, in order to have a perfect match between Equations (32) and (34), the height of centre of gravity $H_{c g}^{*}$ of the model in Figure 4 (left and middle) needs to match the height of the centre of gravity of the multi-mass or equivalent model, defined by $H_{c g}$. However, this is not guaranteed. As a result, the third term in Equations (31) and (34) may not match.

As will be discussed in Section 4, the centre of gravity of the equivalent models are generally lower. Therefore in this paper, different from Yim and Chopra (1985), a new scaling factor is proposed:

$$
\kappa=\frac{H_{c g}}{H_{c g}^{*}}=\frac{m_{t} H_{c g}}{M_{n 1} H_{n 1}}
$$

This factor $\kappa$ accounts for the difference in the height of centre of gravity of the multi mass and equivalent models, and is applied during both the full contact and rocking phases. 


\subsection{Equivalence of equations of motion}

It is useful now to summarize the linearized equilibrium equations of the equivalent model, which is shown in Figure 4 with the parameters identified in Section 3.2. This is only done for the equations which describe the coupled response of first vibration mode and rocking (see Figure 4, left and middle). Equation (32) already described the equations which are used to estimate the higher mode vibration response of multi-mass structures (see Figure 4, right) and these were compared to Equation (20) in Section 3.1.

During the full contact phase, the response of the equivalent model is described by:

$$
\ddot{q}_{n 1}+2 \zeta_{n 1} \omega_{n 1} \dot{q}_{n 1}+\omega_{n 1}^{2} q_{n 1}+\kappa \ddot{u}_{g}=0
$$

Note that this equation is similar to the exact modal component identified in Equation (12). However, the ground motion forcing term in Equation (38) is scaled by an additional parameter $\kappa$. This parameter is the only conceptual difference between Yim and Chopra's (1985) equivalent model and the equivalent model proposed in this paper. The parameter $\kappa$ may result in an overestimation of accelerations due to the first vibration mode. However, as discussed earlier, this conservative approach is necessary to achieve an accurate estimation of the overturning demands due to inertial accelerations in the equivalent model. Without this scaling parameter, it was not possible to achieve good comparison with experimental results, particularly for stocky structures (see Section 4).

Once uplift occurs, the equivalent model simulates combined rocking and vibration action for the first vibration and rocking modes. The linearized equilibrium equations of the equivalent model during the rocking phase can be found by substituting the identified model parameters in Section 3.2 into the general Equations (2) and (3). After neglecting the geometric stiffness terms, this yields:

$$
\begin{gathered}
\ddot{q}_{n 1}+H_{n 1} \ddot{\theta}+2 \zeta_{n 1} \omega_{n 1} \dot{q}_{n 1}+\omega_{n 1}{ }^{2} q_{n 1}+\kappa \ddot{u}_{g}=0 \\
M_{n 1} H_{n 1} \ddot{q}_{n 1}+J_{\mathrm{p}} \ddot{\theta}+m_{t} H_{c g} \ddot{u}_{g} \pm m_{t} g B=0
\end{gathered}
$$

The resulting equations are very similar to Equations (30) and (31), which were derived by simplifying the multi-mass model equilibrium equations. This suggests that an approximate equivalence was established between the identified double lumped mass model and the more complex multi-mass model. This equivalence is expected to be valid as long as the underlying assumptions concerning the modal characteristics of higher modes are valid. These assumptions are evaluated by comparing equivalent model simulations with experimental results and multi-mass simulation results in Section 4. However, a description of the simulation of the equations of motion for the equivalent and multi-mass systems is described first.

\subsection{Numerical simulation}

The equivalent model approximates the multi-mass system as the superposition of a coupled component representing the rocking and first vibration modes (a flexible rocking structure with two lumped masses) and an uncoupled component representing higher vibration modes (SDOF uncoupled oscillators), as schematically illustrated in Figure 4. To determine the equivalent model parameters, Equation (7) is first used to identify the classical modal components of the multi-mass model during full contact phase. These are sufficient for simulating the response of the $2^{\text {nd }}$ and higher vibration modes of the equivalent model. The classical modal characteristics determined from Equation (7) are also used to identify the 
input parameters of the coupled component of the equivalent model using the equations provided in Section 3.2.

Having identified the parameters of the equivalent model, its earthquake response is then determined by numerically solving the nonlinear equations of motion which govern its behaviour. It is noteworthy that the coupled component of the equivalent model is a special case of the general multi mass model. Therefore to determine its earthquake response, the general equations of motion of the multi-mass system can be used (Acikgoz and DeJong 2016). The transition between different phases of motion and the simulation of impact forces using a Dirac-Delta model is adopted for the equivalent model. The optimal parameters identified for the multi-mass models for phase transitions are also utilized for the coupled components of the equivalent model (Acikgoz and DeJong 2016). The uncoupled higher mode responses of the equivalent model are evaluated numerically using Equation (32). These are then superposed on the coupled first vibration and rocking mode response to determine the total response of the equivalent model.
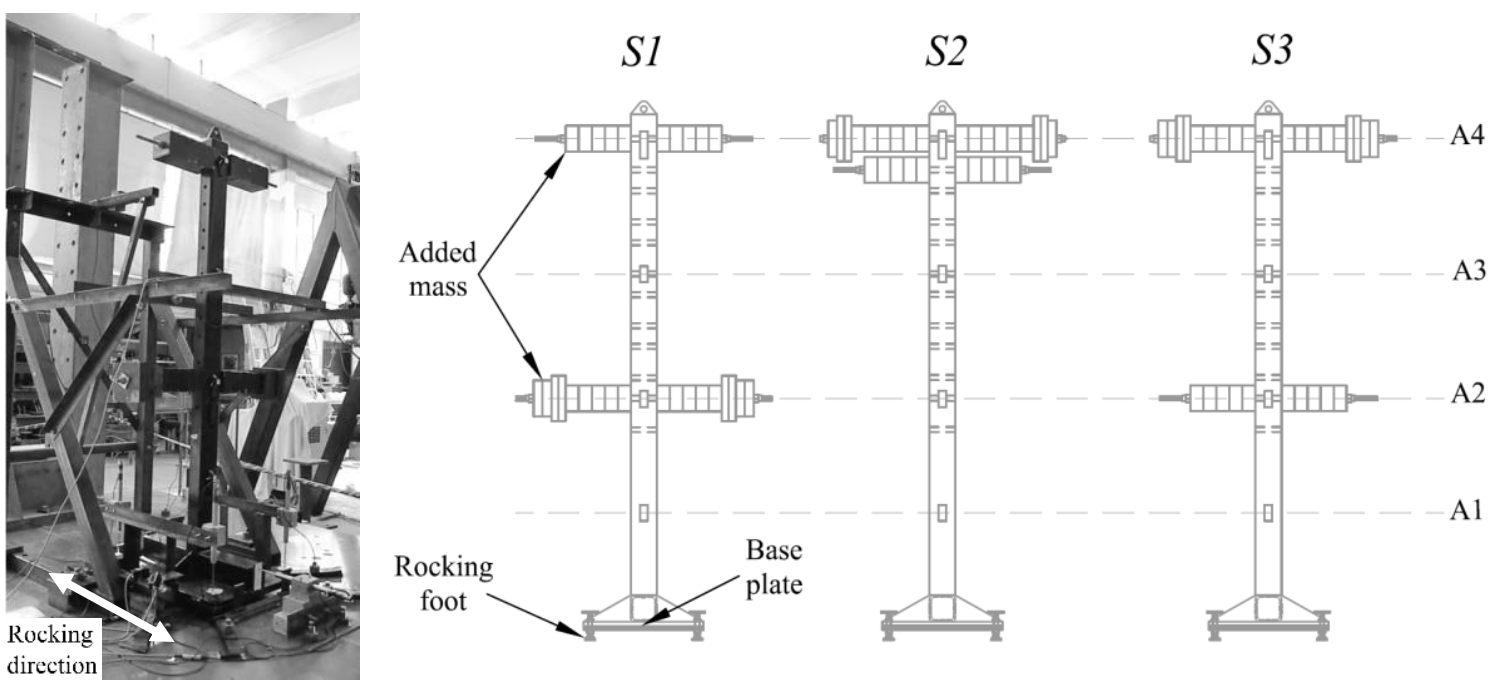

Figure 5 - A photo (left) and elevation drawings (right) of the experimental model illustrating the location of the accelerometers A1-A4 and the mass arrangements S1, S2 and S3.

\section{EVALUATION OF THE EQUIVALENT MODEL}

In this section, experimental tests which are used to evaluate the analytical models are first introduced. Then, multi-mass and equivalent analytical models of the experimental specimens, created using the formulations in Section 2 and 3, are presented. The modal components identified from the multi-mass model are investigated to evaluate the fundamental assumptions concerning the derivation of equivalent models. Then, the equivalent models are used to simulate rocking and acceleration response for free vibration, pulse and earthquake excitation tests. 
Table 1 - Parameters for the multi-mass and equivalent models of the experimental specimens.

\begin{tabular}{|c|c|c|c|c|c|c|}
\hline Specimen \# & 1 & 1 & 2 & 2 & 3 & 3 \\
\hline Model type & Multi-mass & Equivalent & Multi-mass & Equivalent & Multi-mass & Equivalent \\
\hline \multicolumn{7}{|c|}{ Geometric characteristics } \\
\hline$B(\mathrm{~m})$ & 0.305 & 0.305 & 0.305 & 0.305 & 0.305 & 0.305 \\
\hline \multirow{5}{*}{$\underline{H}(\mathrm{~m})$} & 0.06 & \multirow[t]{5}{*}{1.53} & 0.06 & \multirow[t]{5}{*}{1.85} & 0.06 & \multirow[t]{5}{*}{1.76} \\
\hline & 0.49 & & 0.49 & & 0.49 & \\
\hline & 0.93 & & 0.93 & & 0.93 & \\
\hline & 1.43 & & 1.43 & & 1.43 & \\
\hline & 1.93 & & 1.89 & & 1.93 & \\
\hline$H_{c g}(\mathrm{~m})$ & 0.84 & & 1.18 & & 1.02 & \\
\hline$H_{c g}^{*}(\mathrm{~m})$ & & 0.75 & & 1.14 & & 0.95 \\
\hline$\alpha_{c g}(\mathrm{rad})$ & 0.35 & 0.39 & 0.25 & 0.26 & 0.29 & 0.31 \\
\hline \multicolumn{7}{|c|}{ Mass characteristics } \\
\hline \multirow[t]{5}{*}{$\underline{M}(\mathrm{~kg})$} & 77.3 & \multirow[t]{5}{*}{111} & 77.3 & \multirow[t]{5}{*}{139.5} & 77.3 & \multirow[t]{5}{*}{122.1} \\
\hline & 6.2 & & 6.2 & & 6.2 & \\
\hline & 88.2 & & 7.7 & & 48.1 & \\
\hline & 8.2 & & 7.9 & & 8.2 & \\
\hline & 46.2 & & 123.1 & & 86.2 & \\
\hline$m_{0}(\mathrm{~kg})$ & 0 & 115 & 0 & 86.5 & 0 & $\begin{array}{l}103.9 \\
\end{array}$ \\
\hline$J_{0}\left(\mathrm{kgm}^{2}\right)$ & 10 & 17.5 & 10 & 11.9 & 10 & 15.7 \\
\hline$J_{p}\left(\mathrm{kgm}^{2}\right)$ & 298 & 298 & 509 & 509 & 413 & 413 \\
\hline \multicolumn{7}{|c|}{ Vibration characteristics } \\
\hline$\omega_{n 1}(\mathrm{~Hz})$ & 7.6 & 7.5 & 5.2 & 5.2 & 6.0 & 6.0 \\
\hline$\zeta_{n 1}(\%)$ & 0.005 & 0.005 & 0.0025 & 0.0025 & 0.005 & 0.005 \\
\hline$\omega_{n 2}(\mathrm{~Hz})$ & 41.4 & 41.4 & - & - & 50.9 & 50.9 \\
\hline$\zeta_{n 2}(\%)$ & 0.015 & 0.015 & - & - & 0.015 & 0.015 \\
\hline$p_{l}(\mathrm{rad} / \mathrm{s})$ & 2.51 & 2.37 & 2.26 & 2.23 & 2.35 & 2.26 \\
\hline$\overline{r_{e r}}, \overline{r_{e l}}$ & $0.93,0.87$ & $0.93,0.87$ & $0.98,0.93$ & $0.98,0.93$ & $0.94,0.91$ & $0.94,0.91$ \\
\hline$\sigma_{r}$ & 0.025 & 0.025 & 0.0175 & 0.0175 & 0.025 & 0.025 \\
\hline
\end{tabular}

\subsection{Equivalent models of experimental specimens}

The experimental model is a steel column connected to a foundation plate which rests on a shake table through four 'rocking feet' (see Figure 5). Masses were attached along the height of the column to simulate structures with different geometry and vibration characteristics. The three mass arrangements/specimens that were tested are denoted by S1, S2 and S3. The experimental model was instrumented with displacement sensors and accelerometers which measured the rocking angle and accelerations along the height of the structure respectively. In this paper, only rocking rotation, measured by LVDTs at the base, and mid-height acceleration, measured by accelerometer A2 (see Figure 5), will be discussed. Free vibration, pulse and earthquake tests were carried out on each specimen. Further details regarding the experiments can be found in (Acikgoz et al. 2016) .

A multi-mass structural model was created for each specimen in an earlier study (Acikgoz and DeJong 2016). Table 1 lists the important properties of the structural models for these specimens. Using the multi-mass model characteristics, the input parameters for the equivalent models were derived. The first and second mode vibration frequency and damping ( $\omega_{n 1}, \omega_{n 2}, \zeta_{n 1}$ and $\zeta_{n 2}$ ), and foundation half-width $B$ from the multi-mass models were utilized. The remaining structural parameters were defined to achieve the same mass moment of inertia and inertial accelerations due to ground accelerations, in accordance with Section 3.2. For example, for equivalent model 1, the mass matrix $\mathbf{M}$ and height vector $\underline{H}$ describe the top mass $M_{n 1}$ and its height $H_{n 1}$. These were calculated using Equation (11), 
where the known mass, height and classical first mode vibration shape parameters from the multi-mass model were used. Then, by using Equations (35-36), the parameters for the bottom mass $M_{n 0}$ and $J_{0}$ were determined.

It is informative to investigate the resulting dynamic characteristics of the equivalent models and contrast them with the multi-mass models. For instance, by contrasting the multi-mass and equivalent models of specimen S1, it can be observed that the resulting equivalent model has a lower centre of gravity $H_{c g}^{*}$ than the corresponding multi-mass model which has a centre of gravity $H_{c g}$. This leads to the equivalent model having a lower value for the frequency parameter $p_{l}$. However, in the proposed equivalent model formulation, the significant influence on the lower centre of gravity is counteracted by the ground motion scaling term $\kappa$, which was introduced in Equation 37. For specimen S1, this parameter is calculated as $\kappa=H_{c g} / H_{c g}^{*}=1.12$
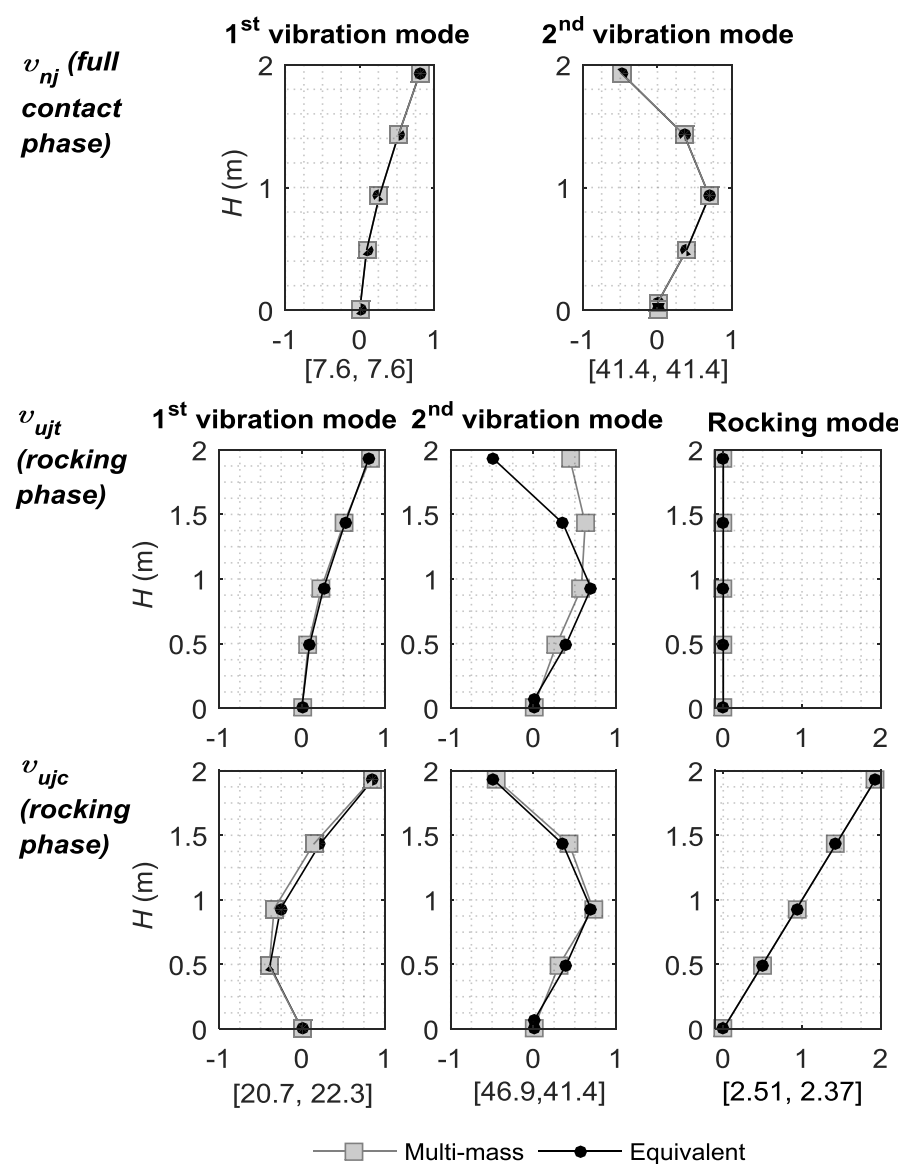

Figure 6 - Modal parameters (frequencies and mode shapes) calculated for the multi-mass and equivalent analytical models of model S1.

\subsection{Modal components of response}

In order to derive equivalent models, important assumptions were made concerning the characteristics of higher vibration modes during rocking. This section assesses the validity of these assumptions, by comparing modal estimations of equivalent models with the modal components of the corresponding multi-mass model. The modal components for the multimass model were determined numerically using Equation (16).

Figure 6 shows the important modal components of response for the analytical models of S1. The sub-figures show the eigenvector estimates for the first, second and 
rocking vibration modes estimated by multi-mass and equivalent models during full contact and rocking phases. The designated values for the 'full contact phase eigenvector' $v_{n j}$ are scaled to Euclidean norm of unity and are shown in the first row. Unsurprisingly, these modal components are the same for the multi-mass and equivalent models during the full contact phase. Under each eigenvector, the associated modal frequencies of multi-mass and equivalent models are presented as $\left[\left(\omega_{k}\right)_{\text {multi-mass }},\left(\omega_{k}\right)_{\text {equivalent }}\right]$ for vibration modes and $\left[\left(p_{l}\right)_{\text {multi-mass }},\left(p_{l}\right)_{\text {equivalent }}\right]$ for the rocking mode.

With the initiation of rocking, the eigen-parameters change. In the second row of Figure 6, the elastic component of the eigenvectors during rocking phase $\underline{v}_{u j t}$ are shown for $j=1,2, r$. Similarly, these are scaled to Euclidean norm of unity and the associated frequencies are listed underneath. The elastic component of the vibration mode shape for the first vibration mode does not change significantly upon the initiation of rocking, and both models compare well. The increase in vibration frequency with the initiation of rocking (listed at the bottom of the third row) is significant, but again the models compare well. On the contrary, for the $2^{\text {nd }}$ vibration mode the modal eigenvector component $\underline{v_{u 2 t}}$ predicted by the two models is significantly different. Furthermore, the multi-mass model predicts a minor increase in the frequency of the second vibration mode, which is not captured by the equivalent model. The third column demonstrates the elastic component of the rocking mode eigenvector. As expected, both the multi-mass and equivalent models estimate negligible elastic component for this mode, confirming that the rocking mode is weakly coupled with elastic response. The identified eigen-parameters, which correspond well to the modified rocking frequency parameter, demonstrate discrepancies due to the different values of $p_{l}$.

The third row of Figure 6 shows the compound mode shapes during rocking. This mode shape takes into account the modal rotation and therefore is critical for determining the force demands on the system. The compound shape is defined by $\underline{v_{u j c}}=\underline{v_{u j t}}+\underline{H} v_{u j r}$ where the rocking component of the mode $\underline{v_{u j r}}$ is added on to the elastic component of the same mode shape $v_{u j t}$. Figure 6 shows that when the effect of this oscillatory rocking component is considered, a drastically different compound shape $\underline{v_{u 1 c}}$ is obtained for the first mode. A nodal point is observed at $H \approx 1.3 \mathrm{~m}$, where eigenvector ordinates change polarity. As both the multi-mass and equivalent models consider the coupling of this mode with rocking action, both models capture the compound shape with good accuracy. Surprisingly, when the effect of the oscillatory rocking component is considered for the second mode, $\underline{v_{u 2 c}}$ becomes similar to the full contact phase eigenvector $\underline{v_{n 2}}$. Therefore, by assuming that the full contact mode shape remains the same during the rocking phase, the equivalent model appears to capture the compound response mode shape. However, this is deceiving. It is important to note that the compound shape of the multi-mass model indicates that there is a rocking component $\underline{v_{u 2 r}}$, which is assumed to be negligible in the equivalent model. Finally, the third column of the third row shows the rocking mode compound shape, which is identical for the multi-mass and equivalent models. 
Table 2 - Important modal characteristics for the multi-mass and equivalent analytical models

\begin{tabular}{|l|l|l|l|l|l|l|l|l|}
\hline Model & $\omega_{u 1}(\mathrm{~Hz})$ & $H_{u 1}(\mathrm{~m})$ & $\chi_{u 1}(-)$ & $\boldsymbol{l}_{u 1}(-)$ & $\omega_{u 2}(\mathrm{~Hz})$ & $H_{u 2}(\mathrm{~m})$ & $\chi_{u 2}(-)$ & $\boldsymbol{t}_{u 2}(-)$ \\
\hline Multi-mass & 20.7 & 1.56 & 0.86 & 1.00 & 46.8 & 1.21 & 0.72 & 0.78 \\
\hline Equivalent & 22.3 & 1.53 & 0.919 & 1.00 & 41.4 & 0.44 & 0 & 0 \\
\hline
\end{tabular}

Broadly, Figure 6 demonstrates that the equivalent model captures the coupling of the first vibration mode and rocking successfully. More importantly, this figure shows that the fundamental assumption of higher vibration mode uncoupling may not be valid for all higher modes. For the examined structure, significant changes are observed in the second vibration mode shape. These findings are further supported by Table 2, where important modal parameters for the first two vibration modes for the multi-mass and equivalent models are compared. These parameters are effective modal height $H_{u k}$ (Equation 11), modal mass participation factor $\chi_{u k}$ (see Equation 22) and force modification parameter $l_{u k}$ (Equation 24). In particular, comparing the effective modal height values $H_{u 2}$ is informative. As a result of the change in the mode shape of the $2^{\text {nd }}$ vibration mode, a high effective modal height of $1.44 \mathrm{~m}$ is observed for the multi-mass model. The equivalent model makes a poor estimate for this modal height by assuming the classical $2^{\text {nd }}$ mode vibration shape during rocking (given by $H_{n 2}$ ) which results in a modal height of $0.44 \mathrm{~m}$. Nonetheless, it is important that this modal height is not zero as assumed for the computation of parameters $\chi_{u 2}$ and $v_{u 2}$ for the equivalent model. Furthermore, the force modification parameter $l_{u 2}$ suggests that neglecting this modal height and coupling could lead to significant consequences. Force modification parameter $l_{u 2}$ values close to unity indicate a strong isolation of this vibration mode from ground motion, but suggest that the mode will be excited at impacts due to the gravity forcing. The significance of these changes in excitation mechanisms will be discussed in the next section.
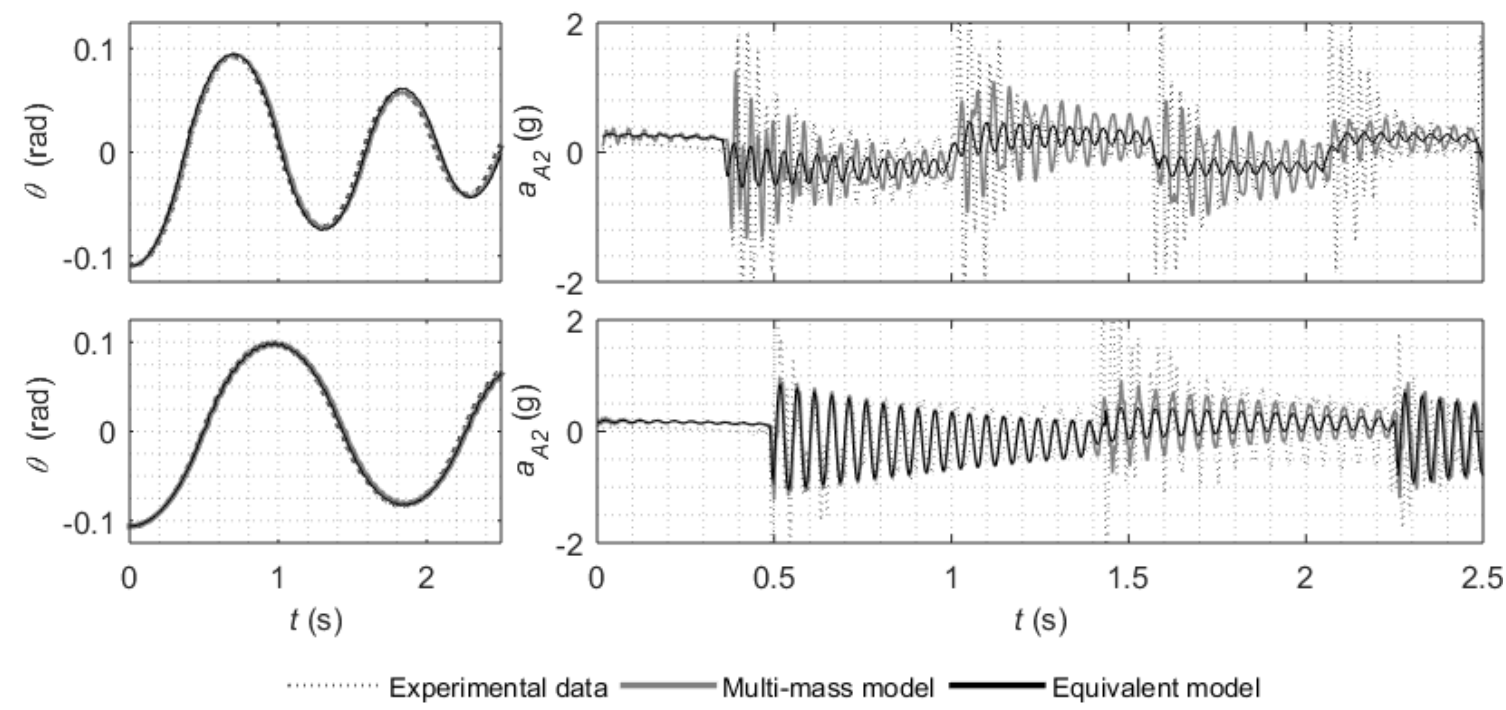

Figure 7 -Comparison of experimental rocking (left) and mid-height acceleration traces (right) with analytical simulations of multi-mass and equivalent models. Specimens S1 (top row) and S2 (bottom row) subjected to free vibration tests are examined.

\subsection{Time history response}

The previous section highlighted that the simplifications adopted in the equivalent models may cause erroneous estimation of rocking and acceleration response. By comparing free 
vibration, pulse and earthquake response simulations of equivalent models with experimental results and multi-mass model simulations, this section quantifies these errors and discusses their significance.

In the free vibration tests, the experimental models were displaced to a large rocking angle and then released with zero initial velocity. For example, Figure 7 shows rocking angle and mid-height acceleration results from large amplitude free vibration tests on S1 and S2. In these tests, the structure is released from a large initial angle of magnitude $0.11 \mathrm{rad}$, which corresponds to approximately $10 \%$ drift. Due to the self-centering provided by the mass, the structure moves towards $\theta=0$, and impacts with the ground. At each impact, a proportion of energy is lost, and a new rocking phase is initiated about the opposite pivot point. In Figure 7 (left), the simulation of rocking traces by equivalent models can hardly be differentiated from the simulation of multi-mass models and experiments. Therefore, the equivalent model is as successful as the multi-mass model in capturing the overall reduction in rocking amplitude and rocking period with time, for both specimens.

The accelerations recorded at mid-height of the structure $a_{\mathrm{A} 2}$ (see Figure 7, right) reveal the clear influence of vibration modes which are excited at impact. When the structure is released from an initial rocking angle in the first rocking half-cycle, only a step acceleration trace is observed. This acceleration step is due to rotational accelerations, which arise as a result of the rigid body motion of the structure. Once impact occurs, the structure starts to rock about the other pivot point, the direction of the step acceleration changes. At the same time, significant vibrations occur. For specimen S1, two components of vibrations with frequencies of approximately $20 \mathrm{~Hz}$ and $40 \mathrm{~Hz}$ form the acceleration signal, particularly just after impact. It is noteworthy that these vibrations are excited without external forcing due to ground motion, and are entirely due to gravity force reversal at impact. For S2, only a single vibration component with a frequency of $20 \mathrm{~Hz}$ is observed. For a detailed examination of these acceleration signals in the frequency domain, see (Acikgoz et al. 2016)
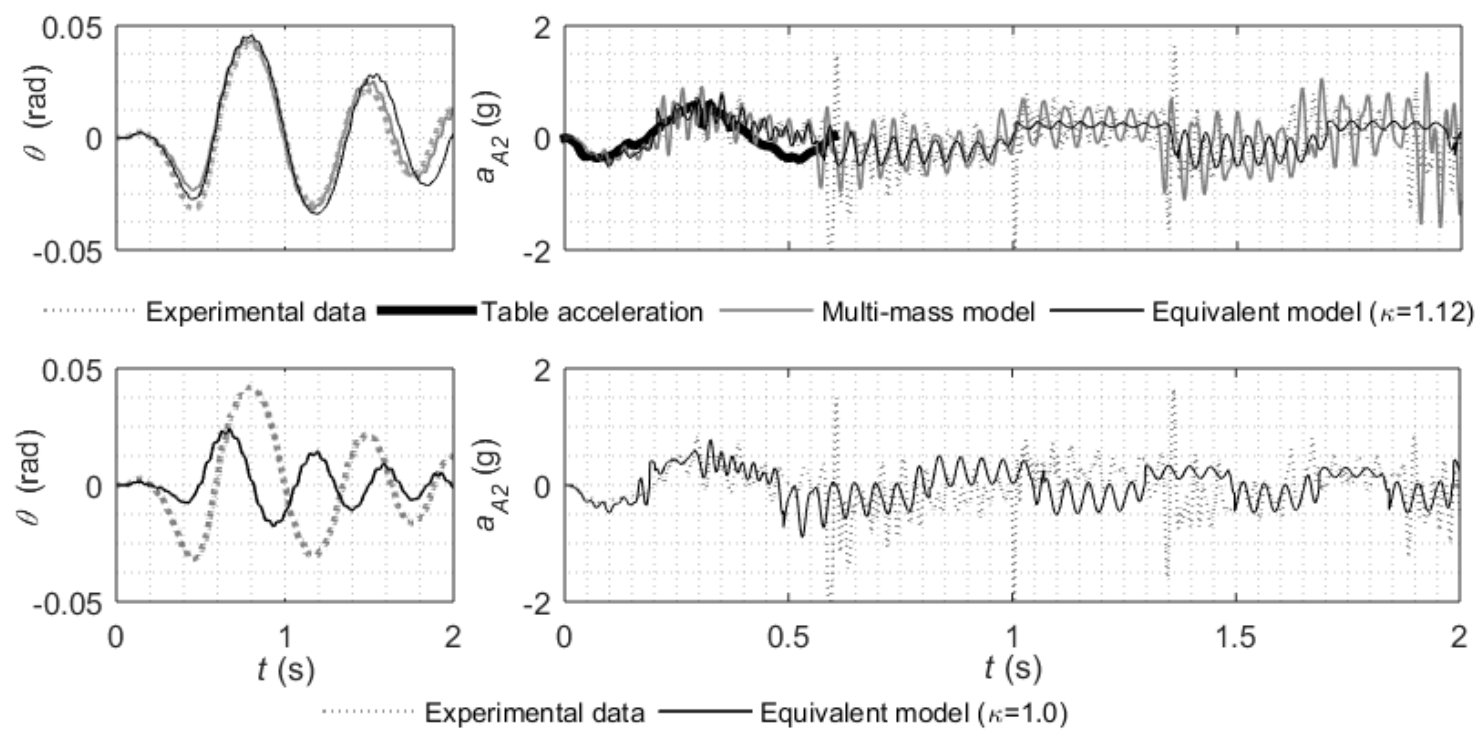

Figure 8 - Comparison of experimental rocking (left) and mid-height acceleration traces (right) with analytical simulations of multi-mass and equivalent models, considering different values of ground motion scaling factor $\kappa$. Specimen S1 subjected to pulse excitation tests is examined.

In general, the characteristics of the acceleration response are simulated well with the multi-mass models, which capture the step profiles as well as the excitation of the first and second vibration modes. The simulations do not capture the large transient acceleration 
spikes which appear for a short duration during impact, but this is not considered significant. These acceleration spikes are related to experimentally observed sliding, bouncing and freeflight effects, and the measurements may have been amplified due to lack of analogue antialiasing filtering of acceleration signals (Acikgoz et al. 2016). Therefore, the following comparisons between experiments and simulations focus on the induced rocking and vibration mode accelerations which have a more obvious structural significance. These comparisons suggest good agreement, which can be further improved by calibrating parameters of the Dirac-delta impact model (Acikgoz and DeJong 2016).

Similarly, the equivalent models capture the step profile and the excitation of the first vibration mode at impacts well. This is evident from the equivalent model simulation of $\mathrm{S} 2$, which is as accurate as the multi-mass analytical model during the half-cycle after the first impact. However, for S1, the equivalent model cannot capture the significant second vibration mode excitation at impact due to the lack of coupling of higher vibration modes and rocking action. This contributes to the significant underestimation of the acceleration demands by the equivalent model and emphasizes an important limitation.

The free vibration response is useful for evaluating the coupling of vibration modes and rocking. However, it is necessary to further evaluate the ability of equivalent models to recognize uplift conditions and to capture the ensuing rocking and acceleration response under pulse and earthquake excitations. In the experimental campaign (Acikgoz et al. 2016), a phased and vertically translated cosine pulse was utilized in all pulse excitation tests. In Figure 8, the experimental rocking and mid-height acceleration response of $\mathrm{S} 1$ to this pulse (defined by $\omega=15 \mathrm{rad} / \mathrm{s}, A=0.4 \mathrm{~g}$ ) is compared to the simulations of multi-mass and various equivalent analytical models. The multi-mass model captures the rocking trace well (see Figure 8, top row), while the rocking predictions depend heavily on the scaling factor $\kappa$ (see Figure 8, bottom row). When a scaling factor is not used (i.e. $\kappa=1$ ), a poor agreement is observed. On the contrary, when the scaling factor $\kappa$ is utilized, the model predictions for rocking action are as accurate as multi-mass models. Therefore, the ground motion scaling factor $\kappa$ specified by Equation (37) will be used for the rest of the equivalent model simulations in this paper. Despite the improvement in rocking angle prediction provided by the scaling factor, the equivalent model underestimates the accelerations for S1 as it cannot capture the higher vibration mode excitation at impact. 

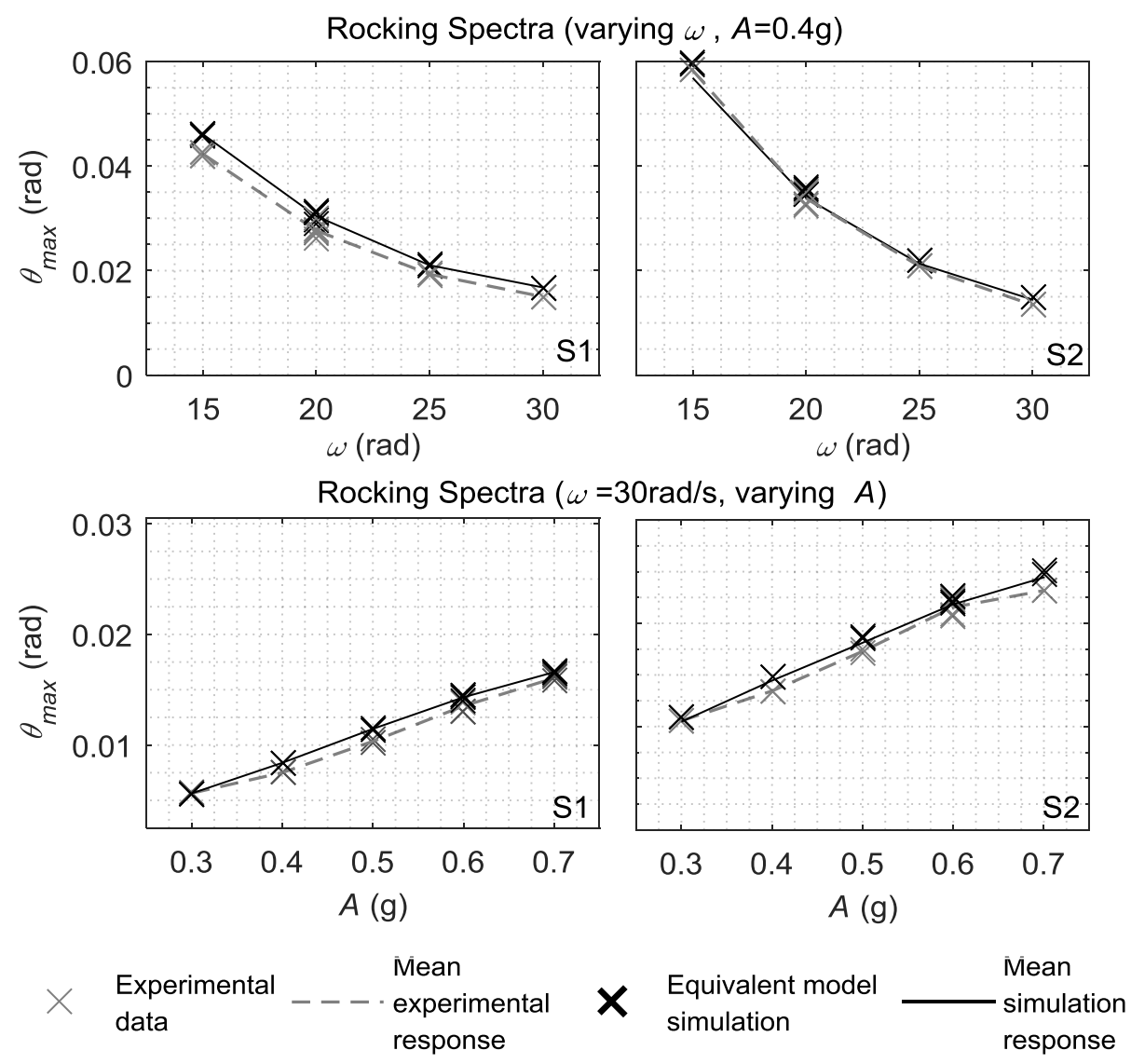

Figure 9 - Comparison of the experimental rocking response spectra to the corresponding spectra simulated by equivalent models. Specimens S1 (left) and S2 (right) subjected to pulse excitation tests of varying frequency (top row) and amplitude (bottom row) are examined.

To generalize the results, it is useful to evaluate the analytical modelling predictions for the maximum rocking $\theta_{\max }$ for a range of pulse excitations. In Figure 9, rocking response spectra compare pulse experiments on S1 and S2 to equivalent model simulations. The first row shows the rocking response spectra of pulses with varying frequency and fixed amplitude. In the second row, the same structure is subjected to pulses with a fixed frequency and varying amplitudes. Each test and simulation was repeated a minimum of two times and the variance of maximum rocking angles in repeated tests was small. The markers in Figure 9 represent individual tests, while the lines represent mean values from the repeated tests. Generally, the equivalent model provides a good prediction of maximum rotation. For both S1 and S2, the predictions lie within 10\% of the experimental observations. 

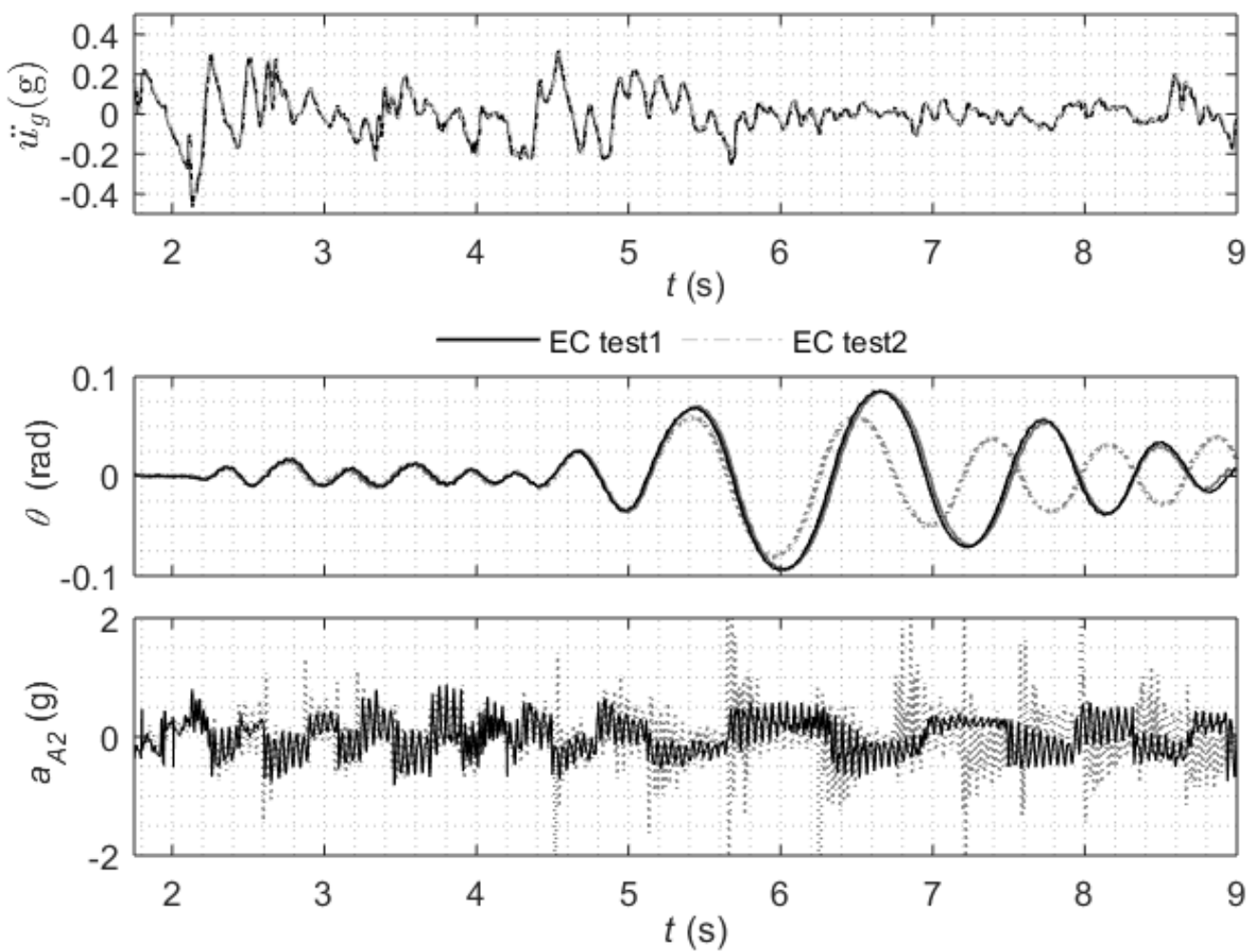

Multi-mass,

Equivalent

increased $r$

(increased $r$ )

$\left(\bar{r}_{r}, \bar{r}_{p}\right)=(0.97,0.91)$

$\left(\bar{r}_{r}, \bar{r}_{p}\right)=(0.97,0.91)$
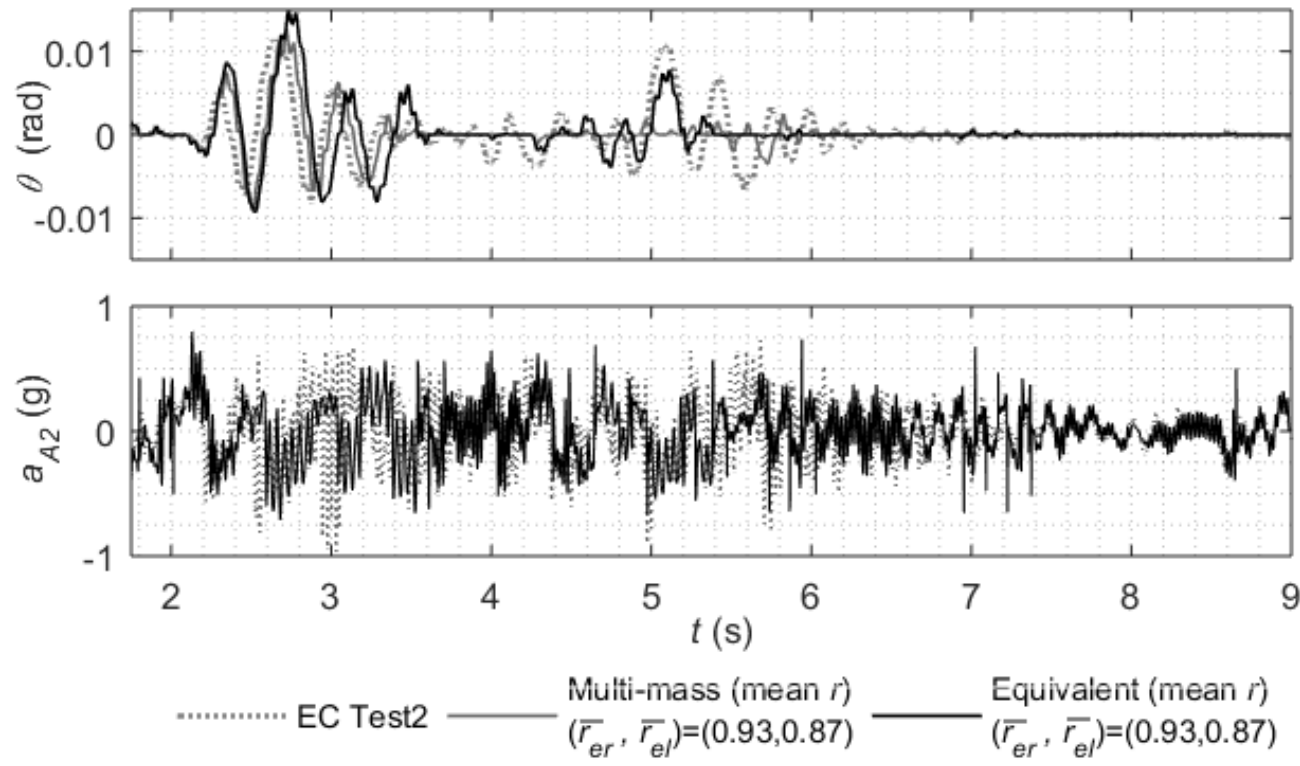

Figure 10 - Comparison of the experimental rocking and mid-height acceleration traces from two earthquake excitation tests with analytical simulations of multi-mass and equivalent models, considering different values of coefficient of restitution $r$. Specimen S1, subjected to repeated earthquake excitation tests (EC Test 1 in first two rows and EC Test 2 in last two rows), is examined.

A similar approach was adopted to investigate the earthquake response experimentally. A single non-pulse type earthquake excitation, scaled to multiple amplitude levels, was considered. The earthquake response to near-identical ground motion excitations resulted in a variable rocking response. The experimental results suggested that the 
variability in response stems from the variability in energy dissipation at impact (Acikgoz et al. 2016). Therefore, the ability of equivalent models to capture the experimentally observed rocking and acceleration response can be evaluated by varying the parameters that define the energy dissipation at impact. For this purpose, the coefficient of restitution $r$ is varied in the simulations. Empirically determined average values for this coefficient (Acikgoz et al. 2016) are shown in Table 1 as $\overline{r_{e r}}$ and $\overline{r_{e l}}$ for impact of the right and left feet of the structure.

Figure 10 shows the rocking and mid-height acceleration response to two amplitude scaled (ground motion scaling factor, $A_{s c}=1.1$ ) El Centro record tests on S1. These tests (EC Test 1 and EC Test2) resulted in drastically different response despite nearly identical table motions (see Figure 10, top row). To simulate EC Test 1 and EC Test 2, multi-mass and equivalent models were first used with coefficients of restitution of $\left(\overline{r_{e r}}, \overline{r_{e l}}\right)=(0.93,0.87)$.

Both models predict a similar response which is in good agreement with the EC Test2 results. By changing the coefficient of restitution to $\left(\bar{r}_{r}, \bar{r}_{l}\right)=(0.97,0.91)$, both analytical models predict EC Test 1 response also with good accuracy (Figure 10, second row). However, for EC Test 1, the response is underdamped after realization of larger amplitudes. Thus, the comparisons indicate that the equivalent model is as good as the multi-mass model in predicting the earthquake response. The major challenge for both models is capturing the variations in energy dissipation.

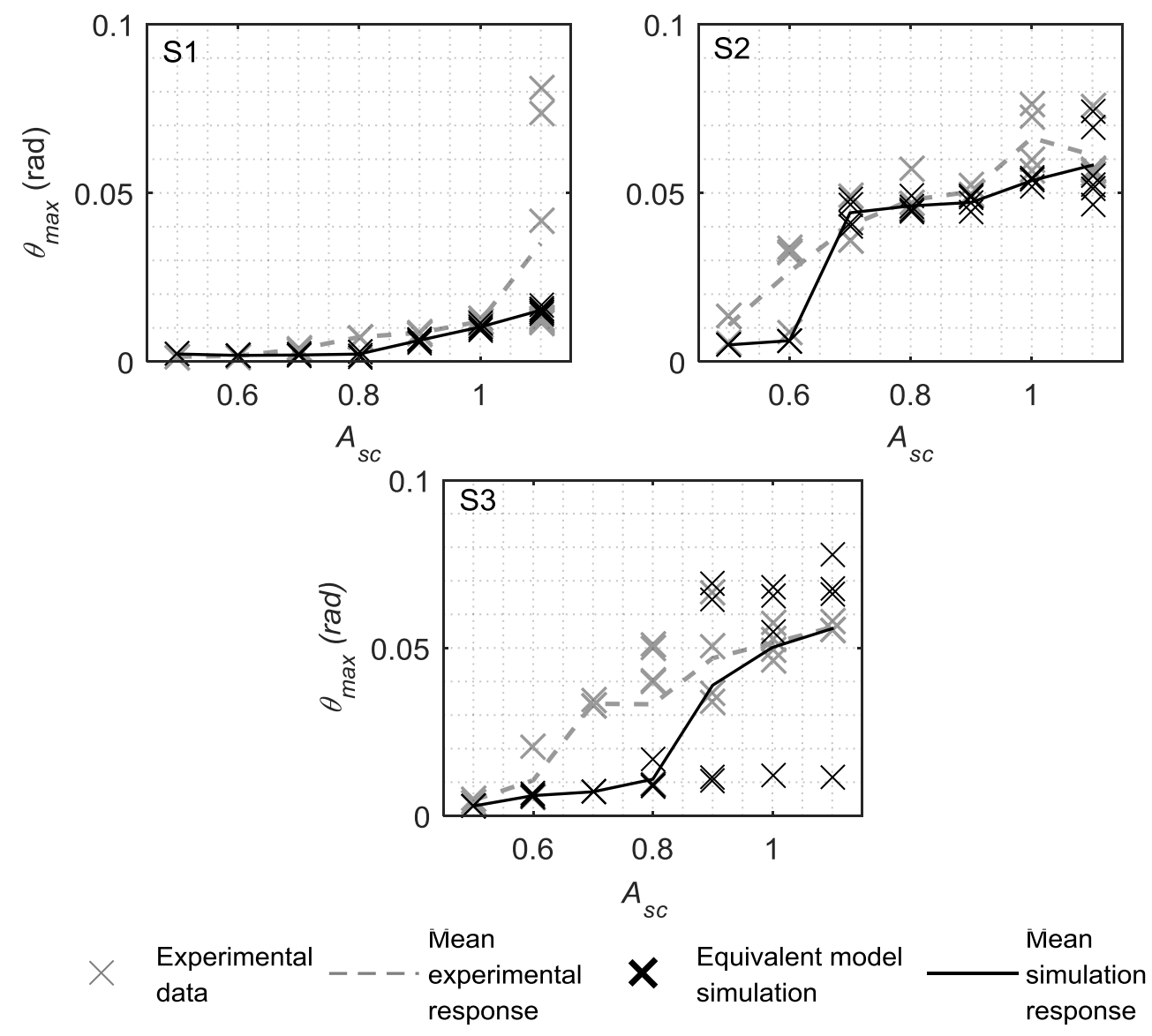

Figure 11 - Comparison of the experimental rocking response spectra to the corresponding spectra simulated by equivalent models. Specimens S1 (top left), S2 (top right) and S3 (bottom) subjected to earthquake excitation tests of varying amplitude scale $A_{s c}$ are examined. 
The acceleration predictions follow similar trends discussed earlier. For clarity, only the equivalent model results are presented in Figure 9. The second vibration mode frequency in the experiments is approximately $40 \mathrm{~Hz}$, and the input ground motion excitation frequencies are insignificant at this level. Therefore, the second and higher vibration mode excitations that are observed in the experimental acceleration traces are primarily caused by impacts. Even if the equivalent model sufficiently captures the overall acceleration trace characteristics and the coupling of the first vibration mode with rocking action, it does not capture the second mode excitation at impact. However, it is noteworthy that this limitation does not affect rocking response predictions, where equivalent model predictions are as good as multi-mass model predictions.

To generalize the results further, more earthquake tests are simulated. To estimate the variability in energy dissipation [25], and therefore better capture the variability of earthquake response, the mean coefficient of restitution $\left(\overline{r_{e r}}, \overline{r_{e l}}\right)$ and standard deviation $\sigma_{r}$ values listed in Table 1were used to assign a random coefficient of restitution for each impact. In Figure 11, the rocking spectral response of all three experimental specimens to the scaled El Centro earthquakes is evaluated. Equivalent models were used with randomly generated coefficients of restitution. Each simulation was repeated a minimum of three times for each amplitude scale using the table motion from each single test. The results for individual experimental tests and equivalent model simulations are presented in the figure. From the individual simulation results for each amplitude scale, a mean spectrum was computed by averaging the rocking response. The mean spectrum is presented for experimental results and equivalent model simulations. In general, the variability in experimental and simulation results is similar, and the trend of increased rocking response with an increase in $A_{s c}$ is captured. It is essential to reiterate that, the equivalent model predictions for rocking are as good as multi-mass models (compare Figure 11 of this paper with Figure 12 of (Acikgoz and DeJong 2016)), and the major modelling limitation concerns the overestimation of energy dissipation during small rocking motion. 

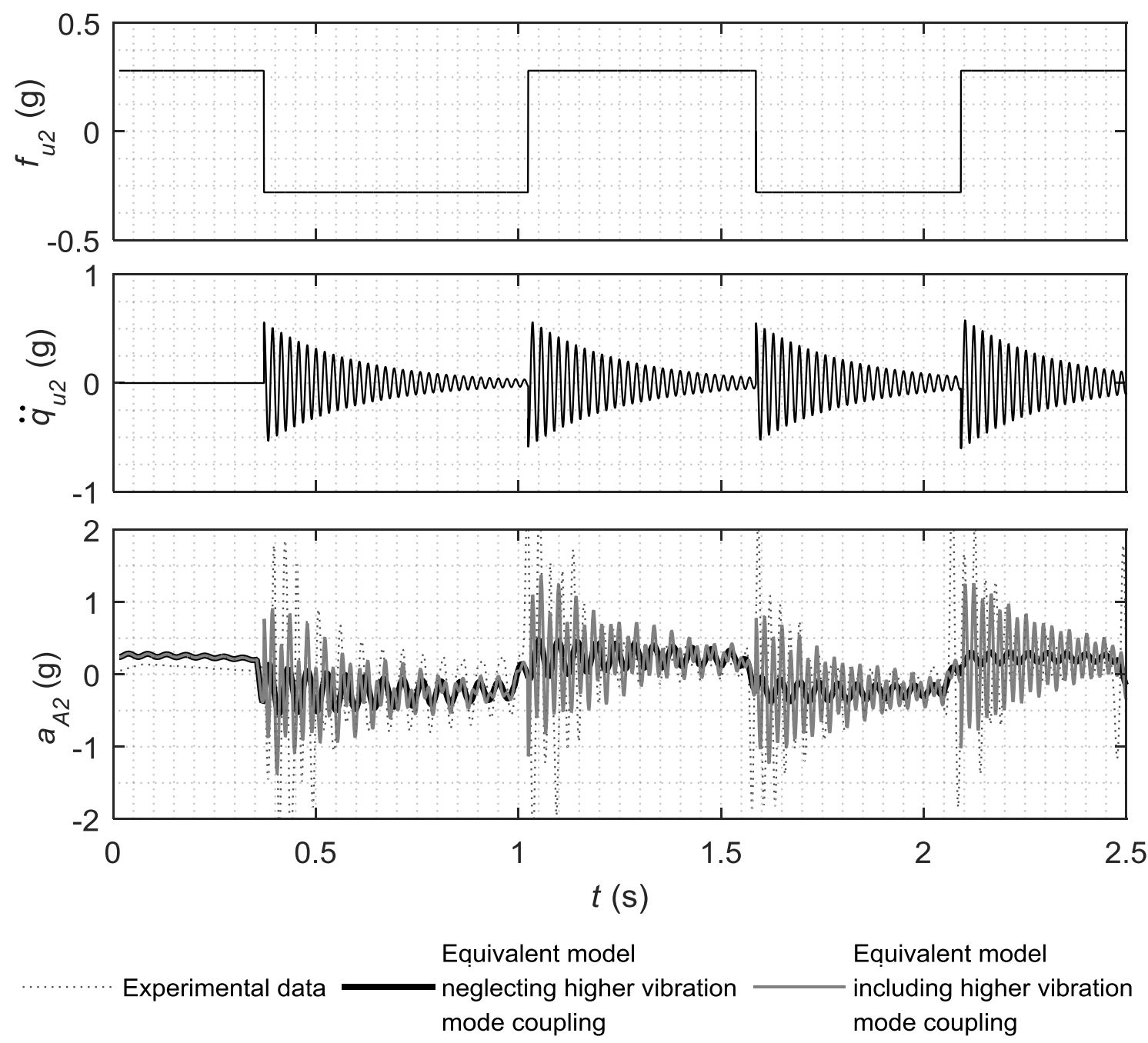

Figure 12 - Improved estimations for modal forcing (top row) and generalized coordinate ( $2^{\text {nd }}$ row) of the second vibration mode and their effect on prediction of acceleration response of S1 in Figure 7 with a modified equivalent model. The legend refers to the data in the bottom row.

\subsection{Improvement of acceleration response predictions}

With the ground motion scaling factor modification proposed in Section 3.2, the original equivalent model, which is conceptually similar to Yim and Chopra's equivalent model (1985) was improved. As demonstrated in Figure 8, and all the figures thereafter, the use of this term enables the equivalent models to adequately capture the rocking response during pulse and earthquake excitations.

However, the assumption of uncoupling of higher vibration modes is not accurate for all vibration modes and resulted in the under-prediction of acceleration demands. To improve the equivalent model predictions of acceleration traces, a new approach to include higher mode coupling effects is briefly proposed in this section. The approach uses acceleration estimates due to the coupled component from the two degree of freedom model as before, but now uses the novel modal equations proposed in this paper (section 2) to estimate the additional contribution from the uncoupled higher vibration modes. In other words, instead of describing higher mode vibration response with linear elastic oscillator responses of classical vibration modes using Equation (32), the behaviour is described using the more accurate Equation (20). This way, the critical excitation of higher vibration modes at impact is considered. More specifically, after identifying the vibration mode parameters during 
rocking from Equation (16), Equation (20) is used to determine acceleration demands due to (i) the ground acceleration, considering isolation and (ii) gravity forcing for a particular mode. To summarise, the only difference between the equivalent model simulations up until this section, and the modified models herein, concern the simulation of higher modes. For simplicity, it will be assumed that the gravity force reversal at impact occurs instantaneously. This will result in the most conservative estimation of induced accelerations. More realistic results could be achieved by exploring the gradual transition between rocking cycles, but this is not necessary for this illustration. The suggested modification is illustrated in Figure 12, where the recorded accelerations for the free vibration test on S1 (presented earlier in Figure 7) are compared to simulation results using the proposed approach. When simulating the accelerations for the earlier Figure 7 with the equivalent model, the uncoupled and SDOF Equation (32) was used. Since there is no ground motion in a free vibration test, this model did not consider any forcing, and the second vibration mode did not contribute to the acceleration response during rocking. In the proposed modification, the modal characteristics during the rocking phase are first computed and are utilized to define the modal forcing defined in Equation (23). This results in a new modal forcing function $f_{u k}$, which is a step function that alternates sign with each rocking half-cycle (see Figure 12, first row). This forcing excites the second vibration mode, whose response is shown in the second row. When this modified response is also included to approximate the total vibration response, a much improved estimation is made for the total acceleration trace, as demonstrated in the final row of Figure 12. However, in some cases, the accelerations are still under-predicted due to the approximate nature of the parameters used to idealize and model impact (Acikgoz and DeJong 2016). Nonetheless the proposed improvement quantifies the significance of the coupling of vibration and rocking and presents a method which could be used for assessment and design to more realistically predict acceleration demands during rocking. It is noteworthy that closed-form solutions (Ayre 1988) may be used to estimate the effect of this simple step forcing on higher vibration modes for design.

\section{CONCLUSIONS}

This paper evaluated and improved simplified equivalent analytical models to describe multi-mass structures rocking on rigid ground. To do this, new equations which describe the modal components of the multi-mass rocking response were derived in Section 2 and the equivalent model simulations were compared to experimental results and multimass model simulations in Section 4.

The findings from the new modal equations provided new insight into the interaction of vibration modes and rocking action and were used to improve the equivalent models. The modal equations quantified the potential changes in the vibration frequencies, shapes and excitation mechanisms once rocking initiates. The changes in the characteristics for the second vibration mode of the experimental specimens, which was neglected by the original equivalent models proposed by Yim and Chopra (1985), was identified as the main reason for the poor modelling of the accelerations by the equivalent model during free vibration tests. In particular, the comparisons demonstrate that it was essential to consider the critical influence of the excitation of higher vibration modes at impact for accurate modelling of acceleration demands, and a method to achieve this is proposed. Similar results would be expected for pulse and earthquake response based on the figures herein and the proposed method also considers the influence of these excitations on acceleration demands. Furthermore, the accuracy of equivalent models in capturing the rocking response was evaluated comprehensively using the results from recent free vibration, pulse and 
earthquake tests. The comparisons reveal that the equivalent models provided excellent estimations of rocking response for a large range of displacements, when a new ground motion amplitude scaling factor was used. This new factor was necessary to compensate for the lower centre of gravity of the equivalent models. With the proposed modifications, the resulting equivalent models provided a simple method to accurately estimate rocking and acceleration response of a complex multi-mass flexible structure rocking on stiff (ideally rigid) ground.

Despite the aforementioned improvements, further research on modelling the complex phenomena at impact and its influence on structural behaviour, is still necessary. In particular, derivation of comprehensive (as well as simplified) analytical models for describing the dynamic response of structures rocking on flexible foundations would be desirable for specific applications.

\section{ACKNOWLEDGEMENTS}

The first author would like to express gratitude for funding from Trinity College, Cambridge Overseas Trust and the Suna and Inan Kirac Foundation.

\section{REFERENCES}

Acikgoz S, Argyle A, DeJong MJ (2014) The role of supplemental damping in limiting forces and displacements in a rocking structure. In: Proceedings of the 2nd European Conference on Earthquake Engineering and Seismology. Istanbul,

Acikgoz S, DeJong M (2013a) Linearization and Modal Analysis of Flexible Rocking Structures. In: New Zealand Society for Earthquake Engineering Conference. Wellington, New Zealand,

Acikgoz S, DeJong MJ (2012) The interaction of elasticity and rocking in flexible structures allowed to uplift. Earthq Eng Struct Dyn 41:2177-2194.

Acikgoz S, DeJong MJ (2013b) Analytical and experimental observations on vibration modes of flexible rocking structures. In: Society of Earthquake and Civil Engineering Dynamics Young Engineers Conference. Newcastle, UK,

Acikgoz S, DeJong MJ (2016) Analytical modelling of a multi-mass flexible rocking structures. Earthq Eng Struct Dyn 45:2103-2122.

Acikgoz S, DeJong MJ (2013c) The rocking response of large flexible structures to earthquakes. Bull Earthq Eng 12:875-908.

Acikgoz S, Ma QT, Palermo A, DeJong MJ (2016) Experimental Identification of the Dynamic Characteristics of a Flexible Rocking Structure. J Earthq Eng 20:1-23.

Ayre RS (1988) Transient response to step and pulse functions. In: Harris CM, Crede CE (eds) Shock and vibration handbook. McGraw-Hill handbooks, p 8.1-8.59

Beck JL, Skinner RI (1973) The seismic response of a reinforced concrete bridge pier designed to step. Earthq Eng Struct Dyn 2:343-358.

Cheng CT (2007) Energy dissipation in rocking bridge piers under free vibration tests. Earthq Eng Struct Dyn 36:503-518.

Chopra AK (2001) Dynamics of structures: theory and applications to earthquake engineering, 2nd edn. Prentice Hall, Upper Saddle River, N.J.

Chopra AK, Yim CS (1985) Simplified earthquake analysis of structures with foundation 
uplift. J Struct Eng 111:2708-2731.

Huckelbridge AA, Clough RW (1978) Seismic response of uplifting building frame. J Struct Div 104:1211-1229.

Kelly JM, Tsztoo DF (1977) Earthquake simulation testing of a stepping frame with energyabsorbing devices. Bull New Zeal Natl Soc Earthq Eng 10:196-207.

Mander JB, Cheng CT (1997) Seismic resistance of bridge piers based on damage avoidance design. Buffalo, NY

Marriott D, Pampanin S, Palermo A (2011) Biaxial testing of unbonded posttensioned rocking bridge piers with external replacable dissipaters. Earthq Eng Struct Dyn 40:1723-1741.

Meek JW (1978) Dynamic Response of Tipping Core Buildings. Earthq Eng Struct Dyn $6: 437-454$.

Meek JW (1975) Effects of foundation tipping on dynamic response. J Struct Div 101:12971311.

Oliveto G, Calio I, Greco A (2003) Large displacement behaviour of a structural model with foundation uplift under impulsive and earthquake excitations. Earthq Eng Struct Dyn 32:369-393.

Palermo A, Pampanin S, Calvi GM (2005) Concept and Development of Hybrid Solutions for Seismic Resistant Bridge Systems. J Earthq Eng 9:899-921.

Pollino M, Bruneau M (2010) Seismic testing of a bridge steel truss pier designed for controlled rocking. J Struct Eng 136:1523-1532.

Priestley MJN, Sritharan S, Conley JR, Pampanin S (1999) Preliminary Results and Conclusions From the PRESSS Five-Story Precast Concrete Test Building. PCI J $44: 42-67$.

Psycharis IN (1991) Effect of base uplift on dynamic response of SDOF structures. J Struct Eng 117:733-754.

Psycharis IN (1983) Dynamics of flexible systems with partial lift-off. Earthq Eng Struct Dyn 11:501-521.

Sharpe RD, Skinner RI (1983) The seismic design of an industrial chimney with rocking base. Bull New Zeal Natl Soc Earthq Eng 16:98-106.

Toranzo LA, Restrepo JI, Mander JB, Carr AJ (2009) Shake-Table Tests of ConfinedMasonry Rocking Walls with Supplementary Hysteretic Damping. J Earthq Eng 13:882-898.

Vassiliou MF, Truniger R, Stojadinovic B (2015) An analytical model of a deformable cantilever structure rocking on a rigid surface: development and verification. Earthq Eng Struct Dyn 44:2795-2815.

Wiebe L, Christopoulos C, Tremblay R, Leclerc M (2012a) Mechanisms to limit higher mode effects in a controlled rocking steel frame. 1: Concept, modelling and lowamplitude shake table testing. Earthq Eng Struct Dyn 42:1053-1068.

Wiebe L, Christopoulos C, Tremblay R, Leclerc M (2012b) Mechanisms to limit higher mode effects in a controlled rocking steel frame. 2: Large-amplitude shake table 
testing. Earthq Eng Struct Dyn 42:1069-1086.

Yim CS, Chopra AK (1985) Simplified earthquake analysis of multistory structures with foundation uplift. J Struct Eng 111:2708-2731. 